ESAIM: PROCEEDINGS, September 2012, Vol. 37, p. 136-165

E. Cancès and S. Labbé, Editors

\title{
BOUNDARY LAYER CORRECTORS AND GENERALIZED POLARIZATION TENSOR FOR PERIODIC ROUGH THIN LAYERS. A REVIEW FOR THE CONDUCTIVITY PROBLEM.
}

\author{
Clair Poignard ${ }^{1}$
}

\begin{abstract}
We study the behaviour of the steady-state voltage potential in a material composed of a two-dimensional object surrounded by a rough thin layer and embedded in an ambient medium. The roughness of the layer is supposed to be $\varepsilon^{\alpha}$-periodic, $\varepsilon$ being the magnitude of the mean thickness of the layer, and $\alpha$ a positive parameter describing the degree of roughness. For $\varepsilon$ tending to zero, we determine the appropriate boundary layer correctors which lead to approximate transmission conditions equivalent to the effect of the rough thin layer. We also provide an explicit characterization of the polarization tensor as defined by Capdeboscq and Vogelius in [9]. The present paper revisits the previous works of the author $[11,13,16,17]$, and it also provides new results for the very rough case $\alpha>1$.

Résumé. Dans cet article, nous considérons le problème de conduction dans un domaine bidimensionnel composé d'une fine membrane rugueuse entourant un domaine conducteur, le tout plongé dans un milieu ambiant de conductivité différente. La rugosité de la membrane est supposée $\varepsilon^{\alpha}$-périodique, $\varepsilon$ étant l'épaisseur moyenne de la membrane, et $\alpha$ un paramètre positif décrivant le degré de rugosité. Nous déterminons des correcteurs de couche limite conduisant à la construction de conditions de transmission approchées lorsque le paramètre $\varepsilon$ tend vers zero. Nous donnons aussi une caractérisation explicite du tenseur de polarisation défini par Capdeboscq and Vogelius dans [9]. Cet article revisite des résultats précédents de l'auteur obtenus dans $[11,13,16,17]$, et présente de nouveaux résultats pour le cas très rugueux $\alpha>1$.
\end{abstract}

\section{INTRODUCTION AND HEURISTICS}

This paper is a review of boundary layer correctors and generalized polarization tensors for the steady-state potential in a dielectric material with a rough thin layer. The roughness of the layer is supposed to be $\varepsilon^{\alpha_{-}}$ periodic, and the mean thickness of the layer is of order $\varepsilon, \varepsilon$ being a small positive parameter, and $\alpha$ a positive parameter describing the degree of roughness.

Several papers are devoted to periodically rough boundaries and derivations of equivalent boundary conditions $[1-3,14]$. In a recent article, Basson and Gérard-Varet [6] derived approximate boundary conditions for a boundary with random roughness. The analysis of these previous papers is essentially based on the construction of the so-called "wall law", which is a boundary condition imposed on an artificial boundary inside the domain. The wall law only reflects the large-scale effect of the oscillations. Note also that in chapter 8 of the book [15],

1 Team-Projet MC2, INRIA Bordeaux-Sud-Ouest, Institut de Mathématiques de Bordeaux, CNRS UMR 5251 \& Université de Bordeaux, 351 cours de la Libération, F-3340 Talence, France.

clair.poignard@inria.fr

(C) EDP Sciences, SMAI 2012 
Marchenko and Khrushlov presented equivalent boundary conditions in the very general framework of elliptic operators with even degree using homogenization techniques.

The core of the present paper consists in deriving transmission conditions equivalent to the rough thin layer in the periodic case. Compared to equivalent boundary conditions, the derivation of equivalent transmission conditions leads to several difficulties in the definition of the boundary layer correctors. In particular, a naive approach coming from wall law derivation techniques can lead to ill-posed problems satisfied by the boundary layer correctors. In addition, we aim at deriving accurate global error estimates, valid in the vicinity of the rough layer, by treating in the same way the weakly oscillating thin layer and the very rough one. We also provide explicit characterizations of the so-called polarization tensor defined in $[4,5,9]$. Actually we aim at showing that the derivation of the boundary layer correctors is very efficient since it provides simultaneously an explicit characterization of the polarization tensor and an accurate description of the electric potential in the vicinity of the inhomogeneity, while the variational techniques as used by Capdeboscq and Vogelius [9] provide estimates only far from the roughness. Note however that the framework of [9] is far more general since it deals with any Lebesgue measurable small inclusions, whereas here we consider the particular case of a rough thin layer.

For $\alpha \leq 1$ several results have been obtained for the conductivity problem [11,16,17]. We aim at revisiting these results using a general framework that allows to treat similarly the three cases $\alpha<1, \alpha=1$ and $\alpha>1$. We emphasize that for $\alpha>1$ only weak results have been obtained in [13] by using two-scale convergence techniques, in the sense that no error estimate has been provided for the approximation. In this paper we push forward the analysis by defining the boundary layer corrector for $\alpha>1$ and by proving error estimates for $\alpha \in(0,2)$ : far from the layer, we recover the results proved in [13], while in the neighborhood of the roughness the boundary layer corrector provides an accurate description of the potential.

Notation 1.1. We now detail the notation used throughout the paper.

- All the closed curves are trigonometrically (counterclockwise) oriented.

- We generically denote by $n$ the normal to a closed smooth curve of $\mathbb{R}^{2}$ outwardly directed from the domain enclosed by the curve to the outside.

- Let $\mathcal{C}$ be a curve of $\mathbb{R}^{2}$, and let $u$ be a sufficiently smooth function defined in a tubular neighborhood of C. We define $\left.u\right|_{\mathcal{C}^{ \pm}}$by

$$
\forall x \in \mathcal{C},\left.\quad u\right|_{\mathcal{C}^{ \pm}}(x)=\lim _{t \rightarrow 0^{+}} u(x \pm \operatorname{tn}(x))
$$

and $\left.\partial_{n} u\right|_{\mathcal{C}^{ \pm}}$denotes

$$
\forall x \in \mathcal{C},\left.\quad \partial_{n} u\right|_{\mathcal{C}^{ \pm}}(x)=\lim _{t \rightarrow 0^{+}} \nabla u(x \pm \operatorname{tn}(x)) \cdot n(x)
$$

where $\cdot$ denotes the Euclidean scalar product of $\mathbb{R}^{2}$.

- The jump $[u]_{\mathcal{C}}$ of a function u defined in a neighborhood of the curve $\mathcal{C}$ is given by

$$
[u]_{\mathcal{C}}=\left.u\right|_{\mathcal{C}^{+}}-\left.u\right|_{\mathcal{C}^{-}}
$$

Let $\Omega$ be a smooth bounded domain of $\mathbb{R}^{2}$ with connected boundary $\partial \Omega$. Let $\varepsilon>0$ and $\alpha>0$ be small positive parameters. We split $\Omega$ into three subdomains: $\mathcal{D}^{1}, \mathcal{D}_{\varepsilon}^{m}$ and $\mathcal{D}_{\varepsilon}^{0}$. The domain $\mathcal{D}^{1}$ is a smooth domain strictly embedded in $\Omega$ (see figure 1), and we denote by $\Gamma$ its boundary, which is a connected smooth curve.

By convention the length of $\Gamma$ equals 1 . Parameterize $\Gamma$ by its curvilinear abscissa denoted by $\Psi$. Let $\kappa$ be the curvature of $\Gamma$ and $n$ its normal vector. Let $f$ be a smooth 1 -periodic and positive function. Note that this assumption can be weakened, after appropriate modifications in the following analysis. Moreover for sake of simplicity, we suppose that $1 / 2 \leq f \leq 3 / 2$. The domain $\mathcal{D}_{\varepsilon}^{m}$ is a thin oscillating layer surrounding $\mathcal{D}^{1}$. We 


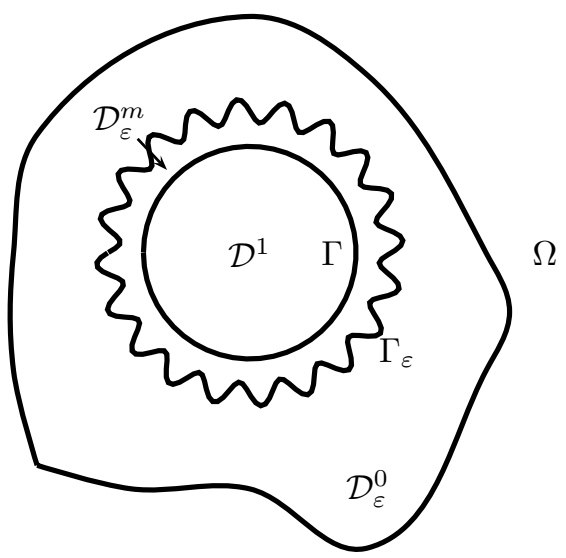

FIGURE 1. Geometry of the problem

denote by $\Gamma_{\varepsilon}^{\alpha}$ the oscillating boundary ${ }^{1}$ of $\mathcal{D}_{\varepsilon}^{m}$ :

$$
\Gamma_{\varepsilon}^{\alpha}=\partial \mathcal{D}_{\varepsilon}^{m} \backslash \Gamma=\left\{\Psi(\theta)+\varepsilon f\left(\theta / \varepsilon^{\alpha}\right) n(\theta), \quad \theta \in \mathbb{T}\right\} .
$$

The domain $\mathcal{D}_{\varepsilon}^{0}$ is defined by

$$
\mathcal{D}_{\varepsilon}^{0}=\Omega \backslash \overline{\left(\mathcal{D}^{1} \cup \mathcal{D}_{\varepsilon}^{m}\right)} .
$$

We also write

$$
\mathcal{D}^{0}=\Omega \backslash \overline{\mathcal{D}^{1}} .
$$

We define the piecewise-constant functions $\sigma^{\varepsilon}, \sigma: \Omega \rightarrow \mathbb{R}$ by

$$
\sigma^{\varepsilon}(z)=\left\{\begin{array}{l}
\sigma_{1}, \text { if } z \in \mathcal{D}^{1}, \\
\sigma_{m}, \text { if } z \in \mathcal{D}_{\varepsilon}^{m}, \\
\sigma_{0}, \text { if } z \in \mathcal{D}_{\varepsilon}^{0},
\end{array} \quad \sigma(z)=\left\{\begin{array}{l}
\sigma_{1}, \text { if } z \in \mathcal{D}^{1}, \\
\sigma_{0}, \text { if } z \in \mathcal{D}^{0},
\end{array}\right.\right.
$$

where $\sigma_{1}, \sigma_{m}$ and $\sigma_{0}$ are given positive constants. Let $g$ be a sufficiently smooth function on $\partial \Omega$ and denote by $u^{\varepsilon}$ the unique function satisfying

$$
\begin{aligned}
& \nabla \cdot\left(\sigma^{\varepsilon} \nabla u^{\varepsilon}\right)=0, \text { in } \Omega, \\
& \left.u^{\varepsilon}\right|_{\partial \Omega}=g,
\end{aligned}
$$

and by $u^{0}$ the background solution:

$$
\begin{aligned}
& \nabla \cdot\left(\sigma \nabla u^{0}\right)=0, \text { in } \Omega, \\
& \left.u^{0}\right|_{\partial \Omega}=g .
\end{aligned}
$$

For the sake of simplicity we suppose that $g$ is a smooth function, but this assumption can be weakened to $g \in H^{s}(\partial \Omega)$ for $s$ large enough. According to Capdeboscq and Vogelius [9], there exists a matrix $\mathcal{M}_{\alpha}$, the

\footnotetext{
${ }^{1}$ To ensure that $\Gamma_{\varepsilon}^{\alpha}$ is a closed curve the small parameter $\varepsilon^{\alpha}$ must equal $1 / N_{\varepsilon}$ for some $N_{\varepsilon} \in \mathrm{N}$, which tends to infinity as $\varepsilon$ goes to zero.
} 
so-called generalized polarization tensor, which is symmetric definite positive and such that almost everywhere far from the inhomogeneity, which is here the rough thin layer, we have:

$$
u^{\varepsilon}(y)-u^{0}(y)=\varepsilon\left(\sigma_{m}-\sigma_{0}\right) \int_{\Gamma} \mathcal{M}_{\alpha}\left(\begin{array}{c}
\partial_{n} u^{0} \\
\nabla_{\Gamma} u^{0}
\end{array}\right) \cdot\left(\begin{array}{c}
\partial_{n} G \\
\nabla_{\Gamma} G
\end{array}\right)(\cdot, y) \mathrm{d} s+o(\varepsilon)
$$

where $G$ is the Dirichlet function defined by

$$
\left\{\begin{array}{l}
\nabla_{x} \cdot\left(\sigma \nabla_{x} G(x, y)\right)=-\delta_{y}, \text { in } \Omega \\
G(x, y)=0, \quad \forall x \in \partial \Omega .
\end{array}\right.
$$

In this paper we provide an explicit characterization of the polarization tensors for rough thin layers for any $\alpha \geq 0$. The parameter $\alpha$ can be seen as a roughness parameter: for $\alpha=0$, the layer is not rough, for $\alpha=1$ the roughness is of same order of the thickness of the layer, and $\alpha>1$ describes very rough thin layers.

For $\alpha>0$, denoting by $\beta=\min (\alpha, 1)$, we define the boundary layer corrector $\left({ }^{\alpha} \mathrm{A},{ }^{\alpha} \mathrm{a}\right)$ by (14), which explicitly characterizes $\mathcal{M}_{\alpha}$ :

$$
\mathcal{M}_{\alpha}=\varepsilon^{\beta-1}\left(\begin{array}{cc}
\sigma_{0}{ }^{\alpha} \mathrm{a}_{X} & \sigma_{0}^{\alpha} \mathrm{a}_{Y} \\
D_{X}^{2} & D_{Y}^{2}
\end{array}\right)
$$

the vector $D^{2}$ being defined by (29). We emphasize that even for $\alpha<1$ the matrix $\mathcal{M}_{\alpha}$ is of order 1 since, according to estimate (15), the quantities ${ }^{\alpha}$ a and $D^{2}$ are of order $\varepsilon^{1-\beta}$. Note the boundary layer corrector $\left({ }^{\alpha} \mathrm{A},{ }^{\alpha} \mathrm{a}\right)$ will be useful to obtain estimates in a neighborhood of the layer (see theorem 3.1).

The outline of the paper is the following. We first rewrite the problem in appropriate coordinates to make appear clearly the boundary layer corrector. We then derive formally the asymptotic expansion of $u^{\varepsilon}$ at the first order in section 2. This section is the core of the paper since it precisely describes the boundary layer correctors for any $\alpha>0$. Section 3 is devoted to the proof of the error estimates for $\alpha \in(0,2)$, and we also link the first boundary layer corrector and the generalized polarization tensor in this part. We end the paper by providing the leading term of the boundary layer corrector for $\alpha \neq 1$ in section 4 . In particular, for very rough thin layers, using the two-scale limit of the boundary layer corrector we retrieve the characterization of the polarization tensor given by Ciuperca et al. in [13] and an accurate description of the potential near the roughness is also available, as proved by theorem 3.1. In the concluding section we provide a link between the results presented in the paper and these of Capdeboscq and Vogelius presented in Proposition 3 of [10], where the cases of thin parallel rectangle inhomogeneities for two different cases are discussed: the case of very oscillating inhomogeneities and the case of slowly oscillating domains.

\subsection{Heuristics of the derivation of the transmission conditions}

The results provided in the present paper deal with quite general geometry of rough thin layers, as described previously. Since the derivation of the asymptotic analysis is technical, we present now the heuristics of the analysis on a model problem to clarify the purpose. We refer the reader to the paper of Allaire and Amar [3] to understand the difference between the derivation of equivalent boundary conditions and transmission conditions.

As proposed in [3], we consider in this paragraph the geometry of the model problem given by figure 2, where $\mathbb{T}=\mathbb{R} / \mathbb{Z}$. It consists of the cylindrical domain $(-1,1) \times \mathbb{T}$ with null curvature, in order to deal with simple expressions of the involved differential operators and normal vectors. We emphasize the relevance of this model problem since all the analysis to treat the general case will consist of appropriate changes of variables in order to recover the configuration of the model problem.

The normal $n_{0}$ to the curve $\{\eta=0\}$ outwardly directed from $\mathcal{O}_{1}$ to its outside is simply

$$
n_{0}=\left(\begin{array}{l}
1 \\
0
\end{array}\right)
$$




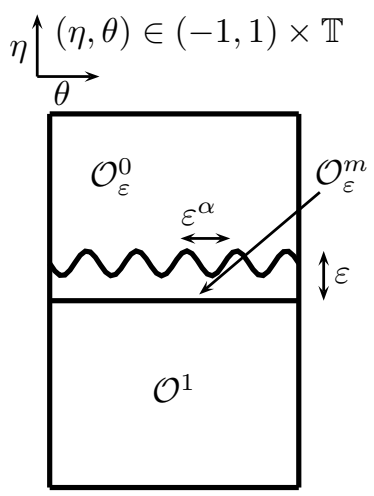

FiguRE 2. Geometry of the model problem

while the normal $n_{\varepsilon}$ to the rough curve $\left\{\left(\varepsilon f\left(\theta / \varepsilon^{\alpha}, \theta\right), \theta \in \mathbb{T}\right\}\right.$ writes

$$
n_{\varepsilon}=\frac{1}{\sqrt{1+\varepsilon^{1-\alpha} f^{\prime}\left(\theta / \varepsilon^{\alpha}\right)}}\left(\begin{array}{c}
1 \\
-\varepsilon^{1-\alpha} f^{\prime}\left(\theta / \varepsilon^{\alpha}\right)
\end{array}\right) .
$$

Therefore, the solution to problem (1) is nothing but the continuous $\theta$-periodic function $u_{\varepsilon}$ that satisfies

$$
\begin{aligned}
& \partial_{\eta}^{2} u_{\varepsilon}+\partial_{\theta}^{2} u_{\varepsilon}=0, \quad \text { in the three domains, } \\
& \left.\sigma_{0}\left(\partial_{\eta} u_{\varepsilon}-\varepsilon^{1-\alpha} f^{\prime}\left(\theta / \varepsilon^{\alpha}\right) \partial_{\theta} u_{\varepsilon}\right)\right|_{\eta^{+}=\varepsilon f\left(\theta / \varepsilon^{\alpha}\right)}=\left.\sigma_{m}\left(\partial_{\eta} u_{\varepsilon}-\varepsilon^{1-\alpha} f^{\prime}\left(\theta / \varepsilon^{\alpha}\right) \partial_{\theta} u_{\varepsilon}\right)\right|_{\eta^{-}=\varepsilon f\left(\theta / \varepsilon^{\alpha}\right)}, \\
& \left.\sigma_{m} \partial_{\eta} u_{\varepsilon}\right|_{\eta=0^{+}}=\left.\sigma_{0} \partial_{\eta} u_{\varepsilon}\right|_{\eta=0^{-}},
\end{aligned}
$$

and the difference $w_{\varepsilon}=u_{\varepsilon}-u_{0}$ is the continuous function that satisfies

$$
\begin{aligned}
& \partial_{\eta}^{2} w_{\varepsilon}+\partial_{\theta}^{2} w_{\varepsilon}=0, \quad \text { in the three domains, } \\
& {\left[\sigma \partial_{n} w_{\varepsilon}\right]_{\eta=\varepsilon f\left(\theta / \varepsilon^{\alpha}\right)}=\left.\left(\sigma_{m}-\sigma_{0}\right) \frac{1}{\sqrt{1+\varepsilon^{1-\alpha} f^{\prime}\left(\theta / \varepsilon^{\alpha}\right)}}\left(\begin{array}{c}
1 \\
-\varepsilon^{1-\alpha} f^{\prime}\left(\theta / \varepsilon^{\alpha}\right)
\end{array}\right) \cdot\left(\begin{array}{c}
\partial_{x} u_{0} \\
\partial_{y} u_{0}
\end{array}\right)\right|_{\eta=\varepsilon f\left(\theta / \varepsilon^{\alpha}\right)},} \\
& {\left[\sigma \partial_{n} w_{\varepsilon}\right]_{\eta=0}=\left.\left(\sigma_{0}-\sigma_{m}\right)\left(\begin{array}{l}
1 \\
0
\end{array}\right) \cdot\left(\begin{array}{c}
\partial_{x} u_{0} \\
\partial_{y} u_{0}
\end{array}\right)\right|_{\eta=0^{+}} .}
\end{aligned}
$$

Since $\left.\left.\nabla u_{0}\right|_{\varepsilon f(. / \varepsilon)} \sim \nabla u_{0}\right|_{0^{+}}$, equation (4) shows that at the order $O(\varepsilon)$ the fast and slow variables are uncoupled. The idea of the asymptotics is to introduce a corrector that takes advantage of this uncoupling. Setting $\beta=\min (1, \alpha)$, we introduce the pair $\left({ }^{\alpha} \mathrm{A},{ }^{\alpha} \mathrm{a}\right)$, where ${ }^{\alpha} \mathrm{A}$ is a vector field of $\mathbb{R} \times \mathbb{T}$ and ${ }^{\alpha} \mathrm{a}$ is a constant vector, defined in the infinite strip $\mathbb{R} \times \mathbb{T}$ by

$$
\begin{aligned}
& \Delta_{X, Y}{ }^{\alpha} \mathrm{A}=0, \text { in } \mathbb{R} \times \mathbb{T} \backslash\left(\mathcal{C}_{0} \cup \mathcal{C}_{\beta ; 1}\right), \\
& \left.\sigma_{0} \nabla_{X, Y}{ }^{\alpha} \mathrm{A}\right|_{\mathcal{C}_{\beta ; 1}^{+}} \cdot n_{\mathcal{C}_{\beta ; 1}}-\left.\sigma_{m} \nabla_{X, Y}{ }^{\alpha} \mathrm{A}\right|_{\mathcal{C}_{\beta ; 1}^{-}} \cdot n_{\mathcal{C}_{\beta ; 1}}=\frac{1}{\sqrt{1+\varepsilon^{1-\alpha} f^{\prime}\left(\theta / \varepsilon^{\alpha-\beta}\right)}}\left(\begin{array}{c}
1 \\
-\varepsilon^{1-\alpha} f^{\prime}\left(\theta / \varepsilon^{\alpha-\beta}\right)
\end{array}\right), \\
& \left.\sigma_{m} \nabla_{X, Y}{ }^{\alpha} \mathrm{A}\right|_{\mathcal{C}_{0}^{+}} \cdot n_{\mathcal{C}_{0}}-\left.\sigma_{1} \nabla_{X, Y}{ }^{\alpha} \mathrm{A}\right|_{\mathcal{C}_{0}^{-}} \cdot n_{\mathcal{C}_{0}}=-\left(\begin{array}{c}
1 \\
0
\end{array}\right), \\
& { }^{\alpha} \mathrm{A} \rightarrow X \rightarrow-\infty 0,{ }^{\alpha} \mathrm{A} \rightarrow X \rightarrow+\infty
\end{aligned}
$$

in the geometry given by figure 3 . 


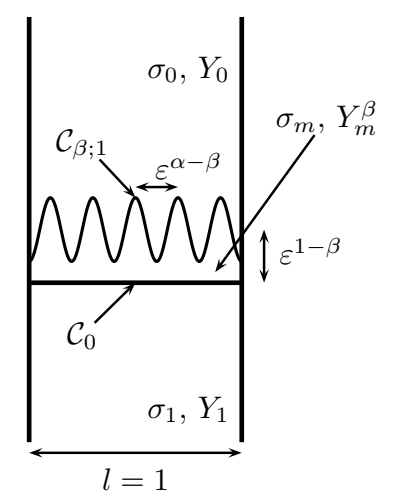

FIGURE 3. Rescaled strip. $\beta=\min (1, \alpha)$.

Then, the function $u_{0}^{B L}$ defined as

$$
u_{B L}^{0}(\eta, \theta)=\left.\left(\sigma_{m}-\sigma_{0}\right) \varepsilon^{\beta} \nabla u^{0}\right|_{0^{+}} \cdot\left\{\begin{array}{l}
\left({ }^{\alpha} \mathrm{A}\left(\eta / \varepsilon^{\beta}, \theta / \varepsilon^{\beta}\right)-{ }^{\alpha}\right), \text { if } \eta>0, \\
{ }^{\alpha} \mathrm{A}\left(\eta / \varepsilon^{\beta}, \theta / \varepsilon^{\beta}\right), \text { if } \eta<0,
\end{array}\right.
$$

should adjust the jumps (4)-(5) at the order $\varepsilon$, but it generates an error on the boundaries $|\eta|=1$ and on the jump of the Dirichlet traces, that are a priori of order $\varepsilon^{\beta}$. We will show that this error is actually smaller, at least for $\alpha<2$ and therefore, that this formal derivation leads to a rigorous approximation of $u_{\varepsilon}$ by $u_{0}+u_{0}^{B L}$. Moreover, we push forward the analysis by providing the approximation at the order $\varepsilon$ in $H^{1}$-norm, which is the energy norm from the physical point of view.

\subsection{The equivalent problem in a tubular neighborhood of $\Gamma$}

It is convenient to write problem (1) in a smooth tubular neighborhood $\Omega_{d_{0}}$ of $\Gamma$ (see figure 4), given for some distance $d_{0}$ such that ${ }^{2}$

$$
d_{0}<\frac{1}{\|\kappa\|_{\infty}}, \text { by } \quad \Omega_{d_{0}}=\left\{z \in \Omega, \quad \operatorname{dist}(z, \Gamma)<d_{0}\right\},
$$

where $\kappa$ is the curvature of $\Gamma$, which is a smooth function of the curvilinear abscissa $\theta$. By definition of $d_{0}$, the normal rays of $\Gamma$ do cross in the domain $\Omega_{d_{0}}$.

Denote by $\Gamma_{-d_{0}}$ and $\Gamma_{d_{0}}$ the closed curves respectively defined by

$$
\Gamma_{-d_{0}}=\partial \Omega_{d_{0}} \cap \mathcal{D}^{1}, \quad \Gamma_{d_{0}}=\partial \Omega_{d_{0}} \cap \mathcal{D}_{\varepsilon}^{0} .
$$

We consider the following Steklov-Poincaré operators $\mathcal{L}_{0}, \mathcal{L}_{1}$ and $\mathscr{T}$ :

$$
\begin{aligned}
& \mathcal{L}_{1}: H^{1 / 2}\left(\Gamma_{-d_{0}}\right) \longrightarrow H^{-1 / 2}\left(\Gamma_{-d_{0}}\right), \\
& \mathcal{L}_{0}: H^{1 / 2}\left(\Gamma_{d_{0}}\right) \longrightarrow H^{-1 / 2}\left(\Gamma_{d_{0}}\right), \\
& \mathscr{T}: H^{1 / 2}(\partial \Omega) \longrightarrow H^{-1 / 2}\left(\Gamma_{d_{0}}\right)
\end{aligned}
$$

${ }^{2}$ We use the notation

$$
\|\kappa\|_{\infty}=\sup _{\theta \in \mathbb{T}}|\kappa(\theta)|
$$




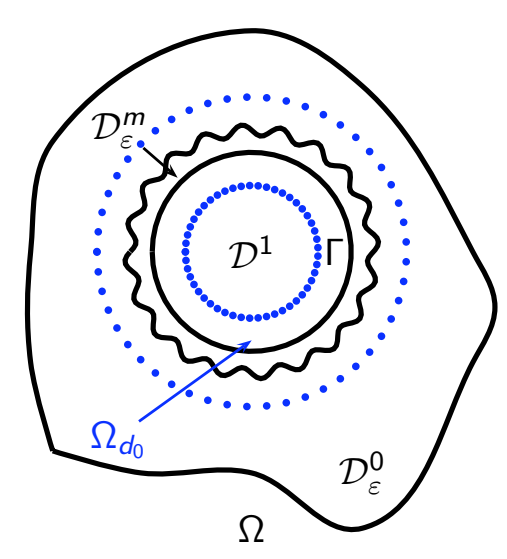

FigurE 4. Tubular neighborhood $\Omega_{d_{0}}$ of the layer

Using the convention of the direction of the normals (see notation 1.1), we define the operator $\mathcal{L}_{1}$ by

$$
\forall \phi \in H^{1 / 2}\left(\Gamma_{-d_{0}}\right), \mathcal{L}_{1}(\phi)=\left.\frac{\partial u}{\partial n}\right|_{\Gamma_{-d_{0}}},
$$

where $u$ is the harmonic function in $\Omega \backslash \overline{\left(\mathcal{D}^{0} \cup \Omega_{d_{0}}\right)}$ equal to $\phi$ on $\Gamma_{-d_{0}}$. The operator $\mathcal{L}_{0}$ is defined by

$$
\forall \phi \in H^{1 / 2}\left(\Gamma_{d_{0}}\right), \mathcal{L}_{0}(\phi)=-\left.\frac{\partial u}{\partial n}\right|_{\Gamma_{d_{0}}},
$$

where $u$ is the harmonic function in $\Omega \backslash \overline{\left(\mathcal{D}^{1} \cup \Omega_{d_{0}}\right)}$ equal to $\phi$ on $\Gamma_{d_{0}}$ and vanishing on $\partial \Omega$. Similarly $\mathscr{T}$ equals:

$$
\forall \chi \in H^{1 / 2}(\partial \Omega), \mathscr{T}(\chi)=-\left.\frac{\partial u}{\partial n}\right|_{\Gamma_{d_{0}}},
$$

where $u$ is the harmonic function in $\Omega \backslash \overline{\left(\mathcal{D}^{1} \cup \Omega_{d_{0}}\right)}$ equal to $\chi$ on $\partial \Omega$ and vanishing on $\Gamma_{d_{0}}$. Moreover, the operators $\mathscr{T}, \mathcal{L}_{0}$ and $\mathcal{L}_{1}$ satisfy the following inequalities, for a $d_{0}$-independent constant $C$ :

$$
\begin{aligned}
& \forall g \in H^{1 / 2}(\partial \Omega), \quad|\mathscr{T} g|_{H^{-1 / 2}\left(\Gamma_{d_{0}}\right)} \leq C|g|_{H^{1 / 2}(\partial \Omega)}, \\
& \forall u \in H^{1 / 2}\left(\Gamma_{d_{0}}\right), \quad\left|\mathcal{L}_{0} u\right|_{H^{-1 / 2}\left(\Gamma_{d_{0}}\right)} \leq C|u|_{H^{1 / 2}\left(\Gamma_{d_{0}}\right)}, \\
& \forall u \in H^{1 / 2}\left(\Gamma_{-d_{0}}\right), \quad\left|\mathcal{L}_{1} u\right|_{H^{-1 / 2}\left(\Gamma_{-d_{0}}\right)} \leq C|u|_{H^{1 / 2}\left(\Gamma_{-d_{0}}\right)},
\end{aligned}
$$

and the following coercivity inequalities hold:

$$
\begin{gathered}
\forall u \in H^{1 / 2}\left(\Gamma_{d_{0}}\right), \quad\left(\mathcal{L}_{0} u, u\right)_{L^{2}\left(\Gamma_{d_{0}}\right)} \geq C|u|_{H^{1 / 2}\left(\Gamma_{d_{0}}\right)}^{2}, \\
\forall u \in H^{1 / 2}\left(\Gamma_{-d_{0}}\right), \quad\left(\mathcal{L}_{1} u, u\right)_{L^{2}\left(\Gamma_{-d_{0}}\right)} \geq C|u|_{H^{1 / 2}\left(\Gamma_{-d_{0}}\right)}^{2} .
\end{gathered}
$$


Problem (1) is then equivalent to

$$
\begin{aligned}
& \nabla .\left(\sigma \nabla u^{\varepsilon}\right)=0, \text { in } \Omega_{d_{0}}, \\
& \left.\partial_{n} u^{\varepsilon}\right|_{\Gamma_{d_{0}}}+\left.\mathcal{L}_{0} u^{\varepsilon}\right|_{\Gamma_{d_{0}}}=-\mathscr{T} g, \text { on } \Gamma_{d_{0}}, \\
& \left.\partial_{n} u^{\varepsilon}\right|_{\Gamma_{-d_{0}}}-\left.\mathcal{L}_{1} u^{\varepsilon}\right|_{\Gamma_{-d_{0}}}=0, \text { on } \Gamma_{-d_{0}} .
\end{aligned}
$$

\subsection{The problem in local coordinates}

The goal of this paragraph is to write problem (1) in the coordinates that live in a domain similar to the domain given in the heuristics of paragraph 1.1 .

Denote by $\Phi$ the smooth diffeomorphism

$$
\forall(\eta, \theta) \in\left(-d_{0}, d_{0}\right) \times \mathbb{T}, \quad \Phi(\eta, \theta)=\Psi(\theta)+\eta n(\theta)
$$

Since $d_{0}<1 /\|\kappa\|_{\infty}$, the open neighborhood of $\Gamma$ denoted by $\Omega_{d_{0}}$ can be parameterized as follows:

$$
\Omega_{d_{0}}=\left\{\Phi(\eta, \theta),(\eta, \theta) \in\left(-d_{0}, d_{0}\right) \times \mathbb{T}\right\}
$$

Let $\mathcal{O}=\left(-d_{0}, d_{0}\right) \times \mathbb{T}$ and denote respectively by $\mathcal{O}^{1}, \mathcal{O}_{\varepsilon}^{m}$ and $\mathcal{O}_{\varepsilon}^{0}$ the domains:

$$
\begin{aligned}
& \mathcal{O}^{1}=\left(-d_{0}, 0\right) \times \mathbb{T}, \\
& \mathcal{O}_{\varepsilon}^{m}=\left\{\left(\eta f\left(\theta / \varepsilon^{\alpha}\right), \theta\right): \quad(\eta, \theta) \in(0, \varepsilon) \times \mathbb{T}\right\}, \\
& \mathcal{O}_{\varepsilon}^{0}=\mathcal{O} \backslash \overline{\mathcal{O}^{1} \cup \mathcal{O}_{\varepsilon}^{m}} .
\end{aligned}
$$

We also denote by $\mathcal{O}^{0}$ the domain $\mathcal{O} \backslash \mathcal{O}^{1}$. Define the oscillating curve $\gamma_{\varepsilon}$ by

$$
\gamma_{\varepsilon}=\left\{\left(\varepsilon f\left(\theta / \varepsilon^{\alpha}\right), \theta\right), \theta \in \mathbb{T}\right\}
$$

We write $\gamma^{s}=\{s\} \times \mathbb{T}$ for any $s \in \mathbb{R}$. The Laplacian written in $(\eta, \theta)$-coordinates equals

$$
\Delta_{\eta, \theta}=\frac{1}{1+\eta \kappa(\theta)} \partial_{\eta}\left((1+\eta \kappa(\theta)) \partial_{\eta}\right)+\frac{1}{1+\eta \kappa(\theta)} \partial_{\theta}\left(\frac{1}{1+\eta \kappa(\theta)} \partial_{\theta}\right)
$$

We also need the normal derivatives on $\Gamma$ and $\Gamma_{\varepsilon}$ in $(\eta, \theta)$-coordinates. In the following, the notation $\nabla_{\eta, \theta}$ denotes the derivative operator:

$$
\nabla_{\eta, \theta}=\left(\begin{array}{cc} 
& \partial_{\eta} \\
(1+\eta \kappa)^{-1} & \partial_{\theta}
\end{array}\right)
$$

Let $u$ be defined on $\Omega$, and define $v$ on $\left(-d_{0}, d_{0}\right) \times \mathbb{T}$ by

$$
\forall(\eta, \theta) \in\left(-d_{0}, d_{0}\right) \times \mathbb{T}, \quad v(\eta, \theta)=u \circ \Phi(\eta, \theta)
$$

We denote by $\partial_{n}^{\Phi} v$ the following normal derivative on $\gamma_{\varepsilon}$ in the local coordinates

$$
\left.\partial_{n}^{\Phi} v\right|_{\gamma_{\varepsilon}}=\left.\nabla_{\eta, \theta} v\right|_{\gamma_{\varepsilon}} \cdot n_{\gamma_{\varepsilon}}
$$


where $n_{\gamma_{\varepsilon}}$ is defined by

$$
n_{\gamma_{\varepsilon}}=\frac{1}{\sqrt{1+\left(\frac{\varepsilon^{1-\alpha} f^{\prime}\left(\theta / \varepsilon^{\alpha}\right)}{1+\varepsilon \kappa f\left(\theta / \varepsilon^{\alpha}\right)}\right)^{2}}}\left(-\frac{\varepsilon^{1-\alpha} f^{\prime}\left(\theta / \varepsilon^{\alpha}\right)}{1+\varepsilon \kappa f\left(\theta / \varepsilon^{\alpha}\right)}\right) .
$$

Moreover, we define the bounded linear operators respectively corresponding to $\mathcal{L}_{0}$ and $\mathcal{L}_{1}$, and denoted by $\Lambda_{0}: H^{1 / 2}\left(\gamma^{d_{0}}\right) \rightarrow H^{-1 / 2}\left(\gamma^{d_{0}}\right)$ and $\Lambda_{1}: H^{1 / 2}\left(\gamma^{-d_{0}}\right) \rightarrow H^{-1 / 2}\left(\gamma^{-d_{0}}\right)$ as follows:

$$
\begin{array}{ll}
\left\langle\Lambda_{0} \varphi, \psi\right\rangle & =\left\langle\mathcal{L}_{0}\left(\varphi \circ \Phi^{-1}\right), \psi \circ \Phi^{-1}\right\rangle, \quad \forall \varphi, \psi \in H^{1 / 2}\left(\gamma^{d_{0}}\right), \\
\left\langle\Lambda_{1} \varphi, \psi\right\rangle & =\left\langle\mathcal{L}_{1}\left(\varphi \circ \Phi^{-1}\right), \psi \circ \Phi^{-1}\right\rangle, \quad \forall \varphi, \psi \in H^{1 / 2}\left(\gamma^{-d_{0}}\right) .
\end{array}
$$

According to (6), there exists an $\varepsilon$-independent constant $C>0$ such that

$$
\begin{array}{ll}
\forall u \in H^{1 / 2}(\mathbb{T}), \quad\left(\Lambda_{0} u, u\right)_{L^{2}(\mathbb{T})} \geq C|u|_{H^{1 / 2}(\mathbb{T})}^{2}, \\
\forall u \in H^{1 / 2}(\mathbb{T}), \quad\left(\Lambda_{1} u, u\right)_{L^{2}(\mathbb{T})} \geq C|u|_{H^{1 / 2}(\mathbb{T})}^{2} .
\end{array}
$$

With this notation, we can write problem (1) in local coordinates. Denoting by $v^{\varepsilon}$ the solution to problem (1) in $(\eta, \theta)$-coordinates,

$$
v^{\varepsilon}=u^{\varepsilon} \circ \Phi .
$$

Then, $v^{\varepsilon}$ is continuous and satisfies

$$
\begin{aligned}
& \Delta_{\eta, \theta} v^{\varepsilon}=0, \text { in } \mathcal{O}^{1} \cup \mathcal{O}_{\varepsilon}^{m} \cup \mathcal{O}_{\varepsilon}^{0}, \\
& \left.\left(1+d_{0} \kappa\right) \partial_{\eta} v^{\varepsilon}\right|_{\eta=d_{0}}+\left.\Lambda_{0} v^{\varepsilon}\right|_{\eta=d_{0}}=-(\mathscr{T} g) \circ \Phi, \\
& \left.\left(1-d_{0} \kappa\right) \partial_{\eta} v^{\varepsilon}\right|_{\eta=-d_{0}}-\left.\Lambda_{1} v^{\varepsilon}\right|_{\eta=-d_{0}}=0
\end{aligned}
$$

with the following transmission conditions:

$$
\begin{aligned}
& \left.\sigma_{0} \partial_{n}^{\Phi} v^{\varepsilon}\right|_{\gamma_{\varepsilon}^{+}}=\left.\sigma_{m} \partial_{n}^{\Phi} v^{\varepsilon}\right|_{\gamma_{\varepsilon}^{-}}, \\
& \left.\sigma_{m} \partial_{\eta} v^{\varepsilon}\right|_{\eta=0^{+}}=\left.\sigma_{1} \partial_{\eta} v^{\varepsilon}\right|_{\eta=0^{-}} .
\end{aligned}
$$

From now on, all the results will be obtained on $v^{\varepsilon}$, but the results for $u^{\varepsilon}$ can be straightforwardly derived using the map $\Phi$.

\subsection{Localization of the roughness}

The derivation of the expansion of $v^{\varepsilon}$ is mainly based on the localization of the oscillations, which leads to solve a boundary value problem in a domain with one oscillation. In order to make appear this "profile" problem, we introduce the following notations. Denote by $\beta=\min (1, \alpha)$. We aim at tackling in the same way all the cases, from the weakly oscillating $(\alpha=0)$ to the very rough cases $(\alpha>1)$. This is the reason why we use the parameter $\beta$ and we perform the rescaling

$$
(\eta, \theta) \rightarrow(X, Y)=\left(\eta / \varepsilon^{\beta}, \theta / \varepsilon^{\beta}\right) .
$$

For sake of simplicity we suppose that the length of $\Gamma$ equals 1 , where $\mathbb{T}=\mathbb{R} / \mathbb{Z}$. Let $\mathcal{C}_{0}$ and $\mathcal{C}_{\beta ; 1}$ be the curves

$$
\mathcal{C}_{0}=\{(0, Y), Y \in \mathbb{T}\}, \quad \mathcal{C}_{\beta ; 1}=\left\{\left(f\left(Y / \varepsilon^{\alpha-\beta}\right), Y\right), Y \in \mathbb{T}\right\}
$$


Denote by $n_{\mathcal{C}_{\beta ; 1}}$ the normal to $\mathcal{C}_{\beta ; 1}$, which is equal on $\mathbb{T}$ to

$$
\forall Y \in \mathbb{T}, \quad n_{\mathcal{C}_{\beta ; 1}}(Y)=\frac{1}{\sqrt{1+\left(\varepsilon^{1-\alpha} f^{\prime}\left(Y / \varepsilon^{\alpha-\beta}\right)\right)^{2}}}\left(\begin{array}{c}
1 \\
-\varepsilon^{1-\alpha} f^{\prime}\left(Y / \varepsilon^{\alpha-\beta}\right)
\end{array}\right),
$$

and let $n_{\mathcal{C}_{0}}$ be the normal to $\mathcal{C}_{0}$ defined by

$$
n_{\mathcal{C}_{0}}(Y)=\left(\begin{array}{l}
1 \\
0
\end{array}\right) \text {. }
$$

It is convenient to denote by $n_{\mathcal{C}_{0}}^{\perp}$ and $n_{\mathcal{C}_{\beta ; 1}}^{\perp}$ the following two unitary vectors:

$$
n_{\mathcal{C}_{0}}^{\perp}(Y)=\left(\begin{array}{l}
0 \\
1
\end{array}\right), \quad n_{\mathcal{C}_{\beta ; 1}}^{\perp}(Y)=\frac{1}{\sqrt{1+\left(\varepsilon^{1-\alpha} f^{\prime}\left(Y / \varepsilon^{\alpha-\beta}\right)\right)^{2}}}\left(\begin{array}{c}
\varepsilon^{1-\alpha} f^{\prime}\left(Y / \varepsilon^{\alpha-\beta}\right) \\
1
\end{array}\right) .
$$

Before performing the asymptotic expansion we would like to make the following remark.

Remark 1.2. According to the definition of $n_{\gamma_{\varepsilon}}$, the slow and the fast variables (respectively the variables $\theta$ and $\theta / \varepsilon^{\alpha}$ ) are mixed in $\left.\partial_{n}^{\Phi} v\right|_{\gamma_{\varepsilon}}$. In order to derive easily the formal asymptotics of the potential, it is convenient to uncouple these variables. Denote by $\mathfrak{f}_{\varepsilon}$ and $M_{\varepsilon}$ the following function

$$
\forall Y \in \mathbb{T}, \quad \mathfrak{f}_{\varepsilon}(Y)=f\left(Y / \varepsilon^{\alpha-\beta}\right), \text { and } \quad M_{\varepsilon}(Y)=\frac{\varepsilon^{1-\alpha} f^{\prime}\left(Y / \varepsilon^{\alpha-\beta}\right)}{\sqrt{1+\left(\varepsilon^{1-\alpha} f^{\prime}\left(Y^{\alpha-\beta}\right)\right)^{2}}} ;
$$

we emphasize that $M_{\varepsilon}$ is uniformly bounded with respect to $\varepsilon$. Using these notations, the normal vector $n_{\gamma_{\varepsilon}}$ writes:

$$
\begin{aligned}
n_{\gamma_{\varepsilon}} & =n_{\mathcal{C}_{\beta ; 1}}\left(\theta / \varepsilon^{\beta}\right)+\varepsilon \kappa \mathfrak{f}_{\varepsilon}\left(\theta / \varepsilon^{\beta}\right)\left[M_{\varepsilon}^{2}\left(\theta / \varepsilon^{\beta}\right) n_{\mathcal{C}_{\beta ; 1}}\left(\theta / \varepsilon^{\beta}\right)+M_{\varepsilon}\left(\theta / \varepsilon^{\beta}\right) n_{\mathcal{C}_{0}}^{\perp}\right] \\
& +O\left(\varepsilon^{2}\right),
\end{aligned}
$$

and for any sufficiently smooth function $\varphi$

$$
\left.\nabla_{\eta, \theta} \varphi\right|_{\gamma_{\varepsilon}}=\left.\nabla_{\eta, \theta} \varphi\right|_{\eta=0}+\left.\varepsilon \mathfrak{f}_{\varepsilon}\left(\theta / \varepsilon^{\beta}\right)\left(\begin{array}{c}
\partial_{\eta}^{2} \varphi \\
-\kappa \partial_{\theta} \varphi+\partial_{\eta} \partial_{\theta} \varphi
\end{array}\right)\right|_{\eta=0^{+}}+O\left(\varepsilon^{2}\right)
$$

Hence, the following two formulae, which will be useful in the derivation of the asymptotic expansion write

$$
\begin{aligned}
\left.\partial_{n}^{\Phi} \varphi\right|_{\gamma_{\varepsilon}} & =\left.\nabla_{\eta, \theta} \varphi\right|_{\gamma_{\varepsilon}} \cdot n_{\mathcal{C}_{\beta ; 1}}\left(\theta / \varepsilon^{\beta}\right) \\
& +\left.\varepsilon \kappa \mathfrak{f}_{\varepsilon}\left(\theta / \varepsilon^{\beta}\right) \nabla_{\eta, \theta} \varphi\right|_{\gamma_{\varepsilon}} \cdot\left[M_{\varepsilon}^{2}\left(\theta / \varepsilon^{\beta}\right) n_{\mathcal{C}_{\beta ; 1}}\left(\theta / \varepsilon^{\beta}\right)+M_{\varepsilon}\left(\theta / \varepsilon^{\beta}\right) n_{\mathcal{C}_{0}}^{\perp}\right] \\
& +O\left(\varepsilon^{2}\right) . \\
\left.\partial_{n}^{\Phi} \varphi\right|_{\gamma_{\varepsilon}} & =\left.\nabla_{\eta, \theta} \varphi\right|_{\eta=0} \cdot n_{\mathcal{C}_{\beta ; 1}}\left(\theta / \varepsilon^{\beta}\right) \\
& +\varepsilon \mathfrak{f}_{\varepsilon}\left(\theta / \varepsilon^{\beta}\right)\left\{\left.\left(\begin{array}{c}
\partial_{\eta}^{2} \varphi \\
\partial_{\eta} \partial_{\theta} \varphi
\end{array}\right)\right|_{\eta=0^{+}} \cdot n_{\mathcal{C}_{\beta ; 1}}\left(\theta / \varepsilon^{\beta}\right)\right. \\
& \left.+\left.\kappa \nabla_{\eta, \theta} \varphi\right|_{\eta=0^{+}} \cdot\left[M_{\varepsilon}^{2}\left(\theta / \varepsilon^{\beta}\right) n_{\mathcal{C}_{\beta ; 1}}\left(\theta / \varepsilon^{\beta}\right)+M_{\varepsilon}\left(\theta / \varepsilon^{\beta}\right) n_{\mathcal{C}_{0}}^{\perp}\right]\right\} \\
& +O\left(\varepsilon^{2}\right) .
\end{aligned}
$$


We emphasize that the terms in $O\left(\varepsilon^{2}\right)$ involve third order derivatives of the function $\varphi$, and they are bounded in $L^{\infty}$ by $\varepsilon^{2}$, up to a multiplicative constant since $\varphi$ is assumed to be smooth enough.

\section{FORMAL ASYMPTOTICS}

\subsection{Zeroth-order approximation}

Let $v^{0}$ be the continuous "background" solution defined by

$$
\begin{aligned}
& \Delta_{\eta, \theta} v^{0}=0, \text { in } \mathcal{O}^{1} \cup \mathcal{O}^{0}, \\
& \left.\left(1+d_{0} \kappa\right) \partial_{\eta} v^{0}\right|_{\eta=d_{0}}+\left.\Lambda_{0} v^{0}\right|_{\eta=d_{0}}=-(\mathscr{T} g) \circ \Phi, \\
& \left.\left(1-d_{0} \kappa\right) \partial_{\eta} v^{0}\right|_{\eta=-d_{0}}-\left.\Lambda_{1} v^{0}\right|_{\eta=-d_{0}}=0, \\
& \left.\sigma_{0} \partial_{\eta} v^{0}\right|_{\eta=0^{+}}=\left.\sigma_{1} \partial_{\eta} v^{0}\right|_{\eta=0^{-}} .
\end{aligned}
$$

Since $g \in \mathcal{C}^{\infty}(\partial \Omega)$, the potential $v^{0}$ belongs to $H^{1}(\Omega)$ and it is smooth in each subdomain $\mathcal{O}^{1}$ and $\mathcal{O}^{0}$.

Denote by $w^{0}$ the error $v^{\varepsilon}-v^{0}$. This continuous function is the unique solution to

$$
\begin{aligned}
& \Delta_{\eta, \theta} w^{0}=0, \text { in } \mathcal{O}^{1} \cup \mathcal{O}^{0}, \\
& \left.\left(1+d_{0} \kappa\right) \partial_{\eta} w^{0}\right|_{\eta=d_{0}}+\left.\Lambda_{0} w^{0}\right|_{\eta=d_{0}}=0, \\
& \left.\left(1-d_{0} \kappa\right) \partial_{\eta} w^{0}\right|_{\eta=-d_{0}}-\left.\Lambda_{1} w^{0}\right|_{\eta=-d_{0}}=0, \\
& \left.\sigma_{0} \partial_{n}^{\Phi} w^{0}\right|_{\gamma_{\varepsilon}^{+}}=\left.\sigma_{m} \partial_{n}^{\Phi} w^{0}\right|_{\gamma_{\varepsilon}^{-}}+\left.\left(\sigma_{m}-\sigma_{0}\right) \partial_{n}^{\Phi} v^{0}\right|_{\gamma_{\varepsilon}}, \\
& \left.\sigma_{m} \partial_{\eta} w^{0}\right|_{\eta=0^{+}}=\left.\sigma_{1} \partial_{\eta} w^{0}\right|_{\eta=0^{-}}+\left.\left(\sigma_{0}-\sigma_{m}\right) \partial_{\eta} v^{0}\right|_{\eta=0^{+}}, \\
& \left.w^{0}\right|_{\partial \Omega}=0, \text { on } \partial \Omega .
\end{aligned}
$$

Since we are interested in the derivation of terms up to order 1, we throw away all the terms, which are $a$ priori of order greater than $\varepsilon$. This approximation will be rigorously justified in the next section for the case $\alpha \in(0,2)$. From the above equality (12), we infer

$$
\left[\sigma \partial_{n}^{\Phi} w^{0}\right]_{\gamma_{\varepsilon}}=\left.\left(\sigma_{0}-\sigma_{m}\right) \nabla_{\eta, \theta} v^{0}\right|_{\eta=0^{+}} \cdot n_{\mathcal{C}_{\beta ; 1}}\left(\theta / \varepsilon^{\beta}\right)+O(\varepsilon) .
$$

Observe also that

$$
\left[\sigma \partial_{n} v^{0}\right]_{\eta=0}=\left.\left(\sigma_{0}-\sigma_{m}\right) \nabla_{\eta, \theta} v^{0}\right|_{\eta=0^{+}} \cdot n_{\mathcal{C}_{0}} .
$$

Therefore, it is natural to introduce a boundary layer corrector in order to correct the Neumann transmission conditions.

\subsubsection{Boundary layer corrector}

We split the infinite strip $\mathbb{R} \times \mathbb{T}$ into three domains:

$$
\begin{aligned}
\mathbb{R} \times \mathbb{T} & =\{X<0, Y \in \mathbb{T}\} \cup\left\{0<X<f\left(Y / \varepsilon^{\alpha-\beta}\right), Y \in \mathbb{T}\right\} \\
\cup & \left\{X>f\left(Y / \varepsilon^{\alpha-\beta}\right), Y \in \mathbb{T}\right\} \cup \mathcal{C}_{0} \cup \mathcal{C}_{\beta ; 1},
\end{aligned}
$$

where $\mathcal{C}_{0}$ and $\mathcal{C}_{\beta ; 1}$ are defined by (10) and let $\sigma^{b}$ be the conductivity corresponding to $\sigma$ in the strip $\mathbb{R} \times \mathbb{T}$ :

$$
\sigma^{b}=\left\{\begin{array}{l}
\sigma_{0}, \quad \text { in } Y_{0}=\left\{(X, Y), \quad X>f\left(Y / \varepsilon^{\alpha-\beta}\right), Y \in \mathbb{T}\right\}, \\
\sigma_{m}, \quad \text { in } Y_{m}^{\beta}=\left\{(X, Y), \quad 0<X<f\left(Y / \varepsilon^{\alpha-\beta}\right), Y \in \mathbb{T}\right\}, \\
\sigma_{1}, \quad \text { in } Y_{1}=\{(X, Y), \quad X<0, Y \in \mathbb{T}\} .
\end{array}\right.
$$




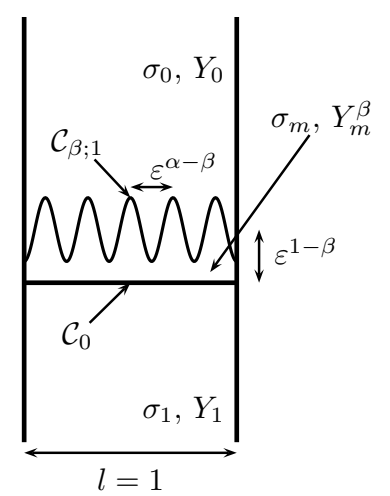

FiguRE 5. Rescaled strip

Observe that the case $\alpha<1(\beta=\alpha)$ is very different from the case $\alpha>1(\beta=1)$ : in the first case, the domain $Y_{m}^{\beta}$ is a thin domain with non constant (but non oscillating) thickness, while in the second case $Y_{m}^{\beta}$ is a domain of measure $O(1)$ with oscillating thickness. These two cases will lead to different leading terms, as it will be proved in section 4 .

Define the pair $\left({ }^{\alpha} \mathrm{A},{ }_{\mathrm{a}}^{\alpha}\right)$, where ${ }^{\alpha} \mathrm{A}$ is a vector field of $\mathbb{R} \times \mathbb{T}$ and ${ }^{\alpha} \mathrm{a}$ is a constant vector, by

$$
\begin{aligned}
& \Delta_{X, Y}{ }^{\alpha} \mathrm{A}=0, \text { in } \mathbb{R} \times \mathbb{T} \backslash\left(\mathcal{C}_{0} \cup \mathcal{C}_{\beta ; 1}\right), \\
& {\left[\left.\sigma^{b} \nabla_{X, Y}{ }^{\alpha} \mathrm{A}\right|_{\mathcal{C}_{\beta ; 1}} \cdot n_{\mathcal{C}_{\beta ; 1}}\right]_{\mathcal{C}_{\beta ; 1}}=n_{\mathcal{C}_{\beta ; 1}},} \\
& {\left[\left.\sigma^{b} \nabla_{X, Y}{ }^{\alpha} \mathrm{A}\right|_{\mathcal{C}_{0}} \cdot n_{\mathcal{C}_{0}}\right]_{\mathcal{C}_{0}}=-n_{\mathcal{C}_{0}},} \\
& { }^{\alpha} \mathrm{A} \rightarrow_{X \rightarrow-\infty} 0,{ }^{\alpha} \mathrm{A} \rightarrow_{X \rightarrow+\infty}{ }^{\alpha} \mathrm{a} .
\end{aligned}
$$

We emphasize that ${ }^{\alpha}$ a is inherent to the problem in the sense that it cannot be imposed as a kind of a Dirichlet condition: the unknowns of the above problem are the vector-field ${ }^{\alpha} \mathrm{A}$ and the constant vector ${ }^{\alpha}$ a. Denote by $\mathscr{E}$ the space of functions defined by

$$
\mathscr{E}=\left\{\begin{array}{l}
\phi \in\left(H_{l o c}^{1}(\mathbb{R} \times \mathbb{T})\right)^{2}: \phi \text { is } y \text {-periodic } \\
\nabla \phi \in L^{2}(\mathbb{R} \times \mathbb{T}) ; \quad \phi \rightarrow_{X \rightarrow-\infty} 0
\end{array}\right\} .
$$

Endowed with the norm

$$
\|\phi\|_{\mathscr{E}}^{2}=\int_{\mathbb{R} \times \mathbb{T}}\|\phi(X, Y)\|^{2} \mathrm{~d} X \mathrm{~d} Y+\int_{\mathbb{R} \times \mathbb{T}}\|\nabla \phi(X, Y)\|^{2} \mathrm{~d} X \mathrm{~d} Y,
$$

$\mathscr{E}$ is a Hilbert space. Since the function $\phi \in \mathscr{E}$ vanishes as $X$ tends to $-\infty$, the semi-norm

$$
\sqrt{\int_{\mathbb{R} \times \mathbb{T}}\|\nabla \phi(X, Y)\|^{2} \mathrm{~d} X \mathrm{~d} Y}
$$

is equivalent to the norm $\|\cdot\|_{\mathscr{E}}$ on $\mathscr{E}$. The pair $\left({ }^{\alpha} \mathrm{A},{ }^{\alpha}\right.$ a) satisfies the following properties. 
Property 2.1. There exists a unique solution $\left({ }^{\alpha} \mathrm{A},{ }^{\alpha} \mathrm{a}\right)$ to problem $(14)$ in $\mathscr{E}$ and there exists an $\varepsilon$-independent constant $C$ such that

$$
\sqrt{\int_{\mathbb{R} \times \mathbb{T}}\left\|\nabla{ }^{\alpha} \mathrm{A}(X, Y)\right\|^{2} \mathrm{~d} X \mathrm{~d} Y} \leq C \varepsilon^{1-\beta}, \quad\left|{ }^{\alpha} \mathrm{a}\right| \leq C \varepsilon^{1-\beta} .
$$

Moreover, the constant vector ${ }^{\alpha}$ a is linked to the vector-field ${ }^{\alpha} \mathrm{A}$ through the following formula:

$$
\begin{aligned}
\sigma_{0}{ }^{\alpha} \mathrm{a} & =\left(\sigma_{0}-\sigma_{m}\right) \int_{0}^{1}{ }^{\alpha} \mathrm{A}\left(\varepsilon^{1-\beta} f\left(Y / \varepsilon^{\alpha-\beta}\right), Y\right) \mathrm{d} Y+\left(\sigma_{m}-\sigma_{1}\right) \int_{0}^{1}{ }^{\alpha} \mathrm{A}(0, Y) \mathrm{d} Y \\
& +\varepsilon^{1-\beta} \int_{0}^{1} f\left(Y / \varepsilon^{\alpha-\beta}\right) \mathrm{d} Y n_{\mathcal{C}_{0} .}
\end{aligned}
$$

Remark 2.2. The $Y$-periodicity and the equations satisfied by ${ }^{\alpha} \mathrm{A}$ imply that ${ }^{\alpha} \mathrm{A}$ decays exponentially fast for $X \rightarrow-\infty$ and similarly ${ }^{\alpha} \mathrm{A}-{ }^{\alpha}$ a decays exponentially fast as $X \rightarrow+\infty$, as described in [11].

Proof. The variational formulation of problem (14) writes:

Find ${ }^{\alpha} \mathrm{A} \in \mathscr{E}$ :

$$
\forall v \in \mathscr{E}, \quad \int_{\mathbb{R} \times \mathbb{T}} \sigma^{b} \nabla^{\alpha} \mathrm{A} \nabla v \mathrm{~d} X \mathrm{~d} Y=\int_{0}^{1} \int_{0}^{\varepsilon^{1-\beta} f\left(Y / \varepsilon^{\alpha-\beta}\right)} \nabla \cdot v(X, Y) \mathrm{d} X \mathrm{~d} Y,
$$

which straightforwardly leads to existence and uniqueness of ${ }^{\alpha} \mathrm{A} \in \mathscr{E}$ and to inequalities (15).

Prove now equality (16). Let $M>2$. By integrating by parts (14), ${ }^{\alpha} \mathrm{A}$ satisfies the following variational formulation:

where $\mathscr{A}$ and $\mathscr{B}$ are given by

$$
\forall v \in\left(H^{1}([-M, M] \times \mathbb{T})\right)^{2}, \quad \mathscr{A}\left({ }^{\alpha} \mathrm{A}, v\right)=\mathscr{B}(v),
$$

$$
\begin{aligned}
\mathscr{A}\left({ }^{\alpha} \mathrm{A}, v\right) & =-\int_{[-M, M] \times \mathbb{T}} \sigma^{b} \nabla^{\alpha} \mathrm{A} . \nabla v \mathrm{~d} X \mathrm{~d} Y+\left.\left.\sigma_{0} \int_{0}^{1} \partial_{X}{ }^{\alpha} \mathrm{A}\right|_{X=M} v\right|_{X=M} \mathrm{~d} Y \\
& -\left.\left.\int_{0}^{1} \sigma_{1} \partial_{X}{ }^{\alpha} \mathrm{A}\right|_{X=-M} v\right|_{X=-M} \mathrm{~d} Y, \\
\mathscr{B}(v) & =\int_{0}^{1} \int_{0}^{\varepsilon^{1-\beta} f\left(Y / \varepsilon^{\alpha-\beta}\right)} \nabla \cdot v(X, Y) \mathrm{d} X \mathrm{~d} Y \\
& =\int_{\mathcal{C}_{1 ; \beta}} n_{\mathcal{C}_{\beta ; 1}}(s) v(s) \mathrm{d} s-\int_{\mathcal{C}_{0}} n_{\mathcal{C}_{0}}(s) v(s) \mathrm{d} s .
\end{aligned}
$$

Moreover, for any function $v \in H^{2}([-M, M] \times \mathbb{T})$, by integrating by parts once again we infer

$$
\begin{aligned}
\mathscr{A}\left({ }^{\alpha} \mathrm{A}, v\right)= & -\left.\sigma_{0} \int_{0}^{1}{ }^{\alpha} \mathrm{A}(M, Y) \partial_{X} v\right|_{M} \mathrm{~d} Y+\left.\sigma_{1} \int_{0}^{1}{ }^{\alpha} \mathrm{A}(-M, Y) \partial_{X} v\right|_{-M} \mathrm{~d} Y \\
& +\left.\sigma_{0} \int_{0}^{1} \partial_{X}{ }^{\alpha} \mathrm{A}(M, Y) v\right|_{M} \mathrm{~d} Y-\left.\sigma_{1} \int_{0}^{1} \partial_{X}{ }^{\alpha} \mathrm{A}(-M, Y) v\right|_{-M} \mathrm{~d} Y \\
& +\int_{\mathcal{C}_{\beta ; 1}}{ }^{\alpha} \mathrm{A}\left[\sigma^{b} \partial_{n} v\right]_{\mathcal{C}_{\beta ; 1}} \mathrm{~d} s+\int_{\mathcal{C}_{0}}{ }^{\alpha} \mathrm{A}\left[\sigma^{b} \partial_{n} v\right]_{\mathcal{C}_{0}} \mathrm{~d} s \\
& +\int_{[-M, M] \times \mathbb{T}} \sigma^{b}{ }^{\alpha} \mathrm{A} \cdot \Delta v \mathrm{~d} X \mathrm{~d} Y .
\end{aligned}
$$


Let $\psi$ be the function defined by

$$
\forall(X, Y) \in(0, M) \times \mathbb{T}, \quad \psi(X, Y)=X
$$

and choose $v=(\psi, \psi)^{T}$ in (17). Denoting by $R_{M}$ the terms

$$
\begin{aligned}
R_{M} & =\sigma_{1} \int_{0}^{1}{ }^{\alpha} \mathrm{A}(-M, Y) \mathrm{d} Y+\sigma_{0} M \int_{0}^{1} \partial_{X}{ }^{\alpha} \mathrm{A}(M, Y) \mathrm{d} Y \\
& +\sigma_{1} M \int_{0}^{1} \partial_{X}{ }^{\alpha} \mathrm{A}(-M, Y) \mathrm{d} Y,
\end{aligned}
$$

we infer that for all $M>2$,

$$
\begin{aligned}
\sigma_{0} \int_{0}^{1}{ }^{\alpha} \mathrm{A}(M, Y) \mathrm{d} Y & =\left(\sigma_{0}-\sigma_{m}\right) \int_{0}^{1}{ }^{\alpha} \mathrm{A}\left(\varepsilon^{1-\beta} f\left(Y / \varepsilon^{\alpha-\beta}, Y\right) \mathrm{d} Y\right. \\
& +\left(\sigma_{m}-\sigma_{1}\right) \int_{0}^{1}{ }^{\alpha} \mathrm{A}(0, Y) \mathrm{d} Y \\
& +\varepsilon^{1-\beta} \int_{0}^{1} f\left(Y / \varepsilon^{\alpha-\beta}\right) \mathrm{d} Y n_{\mathcal{C}_{0}}+R_{M} .
\end{aligned}
$$

Let $M$ tend to infinity to end the proof.

We now define the boundary layer corrector of order $0, v_{B L}^{0}$ on $\mathcal{O}$ by

$$
v_{B L}^{0}(\eta, \theta)=\left.\left(\sigma_{m}-\sigma_{0}\right) \varepsilon^{\beta} \nabla_{\eta, \theta} v^{0}\right|_{\Gamma^{+}} \cdot\left\{\begin{array}{l}
\left({ }^{\alpha} \mathrm{A}\left(\eta / \varepsilon^{\beta}, \theta / \varepsilon^{\beta}\right)-{ }^{\alpha} \mathrm{a}\right), \text { if } \eta>0 \\
{ }^{\alpha} \mathrm{A}\left(\eta / \varepsilon^{\beta}, \theta / \varepsilon^{\beta}\right), \text { if } \eta<0 .
\end{array}\right.
$$

From equality (11) with $\varphi$ replaced by $v_{B L}^{0}$, and according to (15), we deduce the jump of $\partial_{n}^{\Phi} v_{B L}^{0}$ across $\gamma_{\varepsilon}$ :

$$
\begin{aligned}
\frac{1}{\sigma_{m}-\sigma_{0}} & {\left[\sigma \partial_{n}^{\Phi} v_{B L}^{0}\right]_{\gamma_{\varepsilon}}=\left.\nabla_{\eta, \theta} v^{0}\right|_{\eta=0^{+}} \cdot n_{\mathcal{C}_{\beta ; 1}}\left(\theta / \varepsilon^{\beta}\right) } \\
& +\left.\varepsilon^{\beta} \partial_{\theta} \nabla_{\eta, \theta} v^{0}\right|_{\eta=0^{+}} \cdot\left\{\left(\sigma_{m}-\sigma_{0}\right) M_{\varepsilon}(Y)\left(\left.{ }^{\alpha} \mathrm{A}\right|_{\mathcal{C}_{\beta ; 1}}-{ }^{\alpha} \mathrm{a}\right)\right\}_{Y=\theta / \varepsilon^{\beta}} \\
& +\left.\varepsilon \kappa \nabla_{\eta, \theta} v^{0}\right|_{\eta=0^{+}} \cdot\left\{\mathfrak { f } _ { \varepsilon } ( Y ) \left(M_{\varepsilon}^{2}(Y) n_{\mathcal{C}_{\beta ; 1}}(Y)\right.\right. \\
& \left.\left.+M_{\varepsilon}(Y)\left[\sigma^{b} \partial_{Y}{ }^{\alpha} \mathrm{A}\right]_{\mathcal{C}_{\beta ; 1}}\right)\right\}_{Y=\theta / \varepsilon^{\beta}}+O\left(\varepsilon^{2}\right) .
\end{aligned}
$$

In addition, observe that

$$
\begin{aligned}
\left.\partial_{\eta}^{2} v^{0}\right|_{\eta=0^{+}} & =-\left.\kappa \partial_{\eta} v^{0}\right|_{\eta=0^{+}}-\left.\partial_{\theta}^{2} v^{0}\right|_{\eta=0^{+}}, \\
\left.\left(\begin{array}{c}
-\kappa \partial_{\eta} v^{0}-\partial_{\theta}^{2} v^{0} \\
\partial_{\eta} \partial_{\theta} v^{0}
\end{array}\right)\right|_{\eta=0^{+}} \cdot n_{\mathcal{C}_{\beta ; 1}}\left(\theta / \varepsilon^{\beta}\right) & =-\kappa \nabla_{\eta, \theta} v^{0} \cdot n_{\mathcal{C}_{0}} \\
& -\left.\partial_{\theta} \nabla_{\eta, \theta} v^{0}\right|_{\eta=0^{+}} \cdot n_{\mathcal{C}_{\beta ; 1}}^{\perp}\left(\theta / \varepsilon^{\beta}\right)
\end{aligned}
$$


Therefore, using (12)-(18) we infer

$$
\begin{aligned}
{\left[\sigma \partial_{n}^{\Phi}\left(v^{0}+v_{B L}^{0}\right)\right]_{\gamma_{\varepsilon}} } & =\left(\sigma_{m}-\sigma_{0}\right) \varepsilon^{\beta} \partial_{\theta} \nabla_{\eta, \theta} v^{0} \cdot\left[\varepsilon^{1-\beta_{\mathfrak{f}}}(Y) n_{\mathcal{C}_{\beta ; 1}}^{\perp}(Y)\right. \\
& \left.+\left(\sigma_{m}-\sigma_{0}\right) M_{\varepsilon}(Y)\left(\left.{ }^{\alpha} \mathrm{A}\right|_{\mathcal{C}_{\beta ; 1}}-{ }^{\alpha} \mathrm{a}\right)\right]_{Y=\theta / \varepsilon^{\beta}} \\
& +\left.\left(\sigma_{m}-\sigma_{0}\right) \varepsilon^{\beta} \kappa \nabla_{\eta, \theta} v^{0}\right|_{\eta=0^{+}} \cdot\left[\varepsilon ^ { 1 - \beta } \mathfrak { f } _ { \varepsilon } ( Y ) \left(M_{\varepsilon}(Y)\left[\sigma^{b} \partial_{Y}{ }^{\alpha} \mathrm{A}\right]_{\mathcal{C}_{\beta ; 1}}\right.\right. \\
& \left.\left.+n_{\mathcal{C}_{\beta ; 1}}(Y)\right)\right]_{Y=\theta / \varepsilon^{\beta}} \\
& +O\left(\varepsilon^{2}\right) .
\end{aligned}
$$

On the other hand,

$$
\begin{aligned}
\frac{1}{\sigma_{m}-\sigma_{0}} \Delta_{\eta, \theta} v_{B L}^{0}= & \left.\left.\frac{1}{\varepsilon^{\beta}}\left(\frac{1}{(1+\eta \kappa)^{2}}-1\right) \partial_{Y}^{2}{ }^{\alpha} \mathrm{A}\right|_{\eta / \varepsilon^{\beta}, \theta / \varepsilon^{\beta}} \cdot \nabla_{\eta, \theta} v^{0}\right|_{\eta=0^{+}} \\
& +\left.\left.\frac{2}{(1+\eta \kappa)^{2}} \partial_{Y}{ }^{\alpha} \mathrm{A}\right|_{\eta / \varepsilon^{\beta}, \theta / \varepsilon^{\beta}} \cdot \partial_{\theta} \nabla_{\eta, \theta} v^{0}\right|_{\eta=0^{+}} \\
& +\left.\frac{1}{1+\eta \kappa}\left(\left.\kappa \partial_{X}{ }^{\alpha} \mathrm{A}\right|_{\eta / \varepsilon^{\beta}, \theta / \varepsilon^{\beta}}-\left.\frac{\eta \kappa^{\prime}}{(1+\eta \kappa)^{2}} \partial_{Y}{ }^{\alpha} \mathrm{A}\right|_{\eta / \varepsilon^{\beta}, \theta / \varepsilon^{\beta}}\right) \cdot \nabla_{\eta, \theta} v^{0}\right|_{\eta=0^{+}} \\
& +\left.\frac{\varepsilon^{\beta}}{(1+\eta \kappa)^{2}}{ }^{\alpha} \mathrm{A}\right|_{\eta / \varepsilon^{\beta}, \theta / \varepsilon^{\beta}} \cdot\left(\left.\partial_{\theta}^{2} \nabla_{\eta, \theta} v^{0}\right|_{\eta=0^{+}}-\left.\frac{\eta \kappa^{\prime}}{(1+\eta \kappa)^{2}} \partial_{\theta} \nabla_{\eta, \theta} v^{0}\right|_{\eta=0^{+}}\right) .
\end{aligned}
$$

Uncoupling the slow and fast variables, and denoting by $\tilde{O}(\varepsilon)$ any function which is of order $\varepsilon$ as $|\eta| / \varepsilon^{\beta}$ is bounded, and which decays exponentially fast as $|\eta| / \varepsilon^{\beta}$ tends to infinity, we rewrite the previous equality as follows

$$
\begin{aligned}
\frac{1}{\sigma_{m}-\sigma_{0}} \Delta_{\eta, \theta} v_{B L}^{0} & =\left.\left(\partial_{X}{ }^{\alpha} \mathrm{A}-2 X \partial_{Y}^{2}{ }^{\alpha} \mathrm{A}\right)\right|_{(X, Y)=\eta / \varepsilon^{\beta}, \theta / \varepsilon^{\beta}} \cdot\left(\left.\kappa \nabla_{\eta, \theta} v^{0}\right|_{\eta=0^{+}}\right) \\
& +\left.\left.2 \partial_{Y}{ }^{\alpha} \mathrm{A}\right|_{(X, Y)=\eta / \varepsilon^{\beta}, \theta / \varepsilon^{\beta}} \cdot \partial_{\theta} \nabla_{\eta, \theta} v^{0}\right|_{\eta=0^{+}}+\tilde{O}(\varepsilon) .
\end{aligned}
$$

Therefore, $W^{0}=v^{\varepsilon}-\left(v^{0}+v_{B L}^{0}\right)$ satisfies

$$
\begin{aligned}
-\frac{1}{\sigma_{m}-\sigma_{0}} \Delta_{\eta, \theta} W^{0} & =\left.\left(\partial_{X}{ }^{\alpha} \mathrm{A}-2 X \partial_{Y}^{2}{ }^{\alpha} \mathrm{A}\right)\right|_{(X, Y)=\eta / \varepsilon^{\beta}, \theta / \varepsilon^{\beta}} \cdot\left(\left.\kappa \nabla_{\eta, \theta} v^{0}\right|_{\eta=0^{+}}\right) \\
& +\left.\left.2 \partial_{Y}{ }^{\alpha} \mathrm{A}\right|_{(X, Y)=\eta / \varepsilon^{\beta}, \theta / \varepsilon^{\beta}} \cdot \partial_{\theta} \nabla_{\eta, \theta} v^{0}\right|_{\eta=0^{+}}+\tilde{O}(\varepsilon), \\
\frac{1}{\sigma_{m}-\sigma_{0}}\left[\sigma \partial_{n}^{\Phi} W^{0}\right]_{\gamma_{\varepsilon}} & =-\varepsilon^{\beta} \partial_{\theta} \nabla_{\eta, \theta} v^{0} \cdot\left[\left(\sigma_{m}-\sigma_{0}\right) M_{\varepsilon}(Y)\left(\left.{ }^{\alpha} \mathrm{A}\right|_{\mathcal{C}_{\beta ; 1}}-{ }^{\alpha}\right)+\varepsilon^{1-\beta} \mathfrak{f}_{\varepsilon}(Y) n_{\mathcal{C}_{\beta ; 1}}^{\perp}(Y)\right]_{Y=\theta / \varepsilon^{\beta}} \\
& -\left.\varepsilon^{\beta} \kappa \nabla_{\eta, \theta} v^{0}\right|_{\eta=0^{+}} \cdot\left[\varepsilon^{1-\beta} \mathfrak{f}_{\varepsilon}(Y)\left(M_{\varepsilon}(Y)\left[\sigma^{b} \partial_{Y}{ }^{\alpha} \mathrm{A}\right]_{\mathcal{C}_{\beta ; 1}}+n_{\mathcal{C}_{\beta ; 1}}(Y)\right)\right]_{Y=\theta / \varepsilon^{\beta}} \\
& +O\left(\varepsilon^{2}\right) .
\end{aligned}
$$


Denoting by $\left(G^{j}\right)_{j=1,2}$ and $\left(B^{j}\right)_{j=1,2}$ the following terms:

$$
\begin{aligned}
& G^{1}=-\left(\partial_{X}{ }^{\alpha} \mathrm{A}-2 X \partial_{Y}^{2}{ }^{\alpha} \mathrm{A}\right), \quad G^{2}=-2 \partial_{Y}{ }^{\alpha} \mathrm{A}, \\
& B^{1}=-\varepsilon^{1-\beta_{\mathfrak{f}}}\left(M_{\varepsilon}\left[\sigma^{\mathrm{b}} \partial_{Y}{ }^{\alpha} \mathrm{A}\right]_{\mathcal{C}_{\beta ; 1}}+n_{\mathcal{C}_{\beta ; 1}}\right), \\
& B^{2}=-\left[\left(\sigma_{m}-\sigma_{0}\right) M_{\varepsilon}\left(\left.{ }^{\alpha} \mathrm{A}\right|_{\mathcal{C}_{\beta ; 1}}-{ }^{\alpha} \mathrm{a}\right)+\varepsilon^{1-\beta_{\mathfrak{f}_{\varepsilon}}} n_{\mathcal{C}_{\beta ; 1}}^{\perp}\right],
\end{aligned}
$$

we infer

$$
\begin{aligned}
\Delta_{\eta, \theta} W^{0}= & \left.\left(\sigma_{m}-\sigma_{0}\right) \kappa \nabla_{\eta, \theta} v^{0}\right|_{\eta=0^{+}} \cdot G^{1}\left(\eta / \varepsilon^{\beta}, \theta / \varepsilon^{\beta}\right) \\
& +\left.\left(\sigma_{m}-\sigma_{0}\right) \partial_{\theta} \nabla_{\eta, \theta} v^{0}\right|_{\eta=0^{+}} \cdot G^{2}\left(\eta / \varepsilon^{\beta}, \theta / \varepsilon^{\beta}\right)+\tilde{O}(\varepsilon), \\
{\left[\sigma \partial_{n}^{\Phi} W^{0}\right]_{\gamma_{\varepsilon}}=} & \left.\left(\sigma_{m}-\sigma_{0}\right) \varepsilon^{\beta} \kappa \nabla_{\eta, \theta} v^{0}\right|_{\eta=0^{+}} \cdot B^{1}\left(\theta / \varepsilon^{\beta}\right) \\
& +\left(\sigma_{m}-\sigma_{0}\right) \varepsilon^{\beta} \partial_{\theta} \nabla_{\eta, \theta} v^{0} \cdot B^{2}\left(\theta / \varepsilon^{\beta}\right)+O\left(\varepsilon^{2}\right) \\
{\left[\sigma \partial_{n} W^{0}\right]_{\gamma_{0}}=} & 0 \\
{\left[W^{0}\right]_{\gamma_{\varepsilon}}=} & 0 \\
{\left[W^{0}\right]_{\gamma_{0}}=} & \left.\left(\sigma_{m}-\sigma_{0}\right) \varepsilon^{\beta} \cdot \alpha_{\mathrm{a}} \cdot \nabla_{\eta, \theta} v^{0}\right|_{\eta=0^{+}},
\end{aligned}
$$

with the boundary conditions:

$$
\begin{aligned}
& \left.\left(1+d_{0} \kappa\right) \partial_{\eta} W^{0}\right|_{\eta=d_{0}}+\left.\Lambda_{0} W^{0}\right|_{\eta=d_{0}}=-\left.v_{B L}^{0}\right|_{d_{0}}, \\
& \left.\left(1-d_{0} \kappa\right) \partial_{\eta} W^{0}\right|_{\eta=-d_{0}}-\left.\Lambda_{1} W^{0}\right|_{\eta=-d_{0}}=-\left.v_{B L}^{0}\right|_{-d_{0}} .
\end{aligned}
$$

Define $\left(D^{j}\right)_{j=1,2}$ by

$$
D^{j}=\int_{\mathbb{R} \times \mathbb{T}} \sigma^{b} G^{j} \mathrm{~d} X \mathrm{~d} Y+\int_{\mathcal{C}_{\beta ; 1}} B^{j}(s) \mathrm{d} s
$$

Simple calculations imply

$$
D^{2}=\left(\sigma_{m}-\sigma_{0}\right) \int_{\mathcal{C}_{\beta ; 1}} M_{\varepsilon}(s)^{\alpha} \mathrm{A} \mathrm{d} s-\varepsilon^{1-\beta} \int_{0}^{1} f\left(y / \varepsilon^{\alpha-\beta}\right) \mathrm{d} y n_{\mathcal{C}_{0}}^{\perp} .
$$

\subsubsection{Properties of the coefficients $D_{1}$ and $D_{2}$}

Property 2.3. The following properties hold.

(1) The coefficient $D^{1}$ vanishes.

(2) The first component $D_{X}^{2}$ of the vector $D^{2}$ satisfies

$$
D_{X}^{2}=\sigma_{0}{ }^{\alpha} a_{Y},
$$

where the index $X$ (resp. $Y$ ) denotes the first (resp. the second) component of the corresponding vector.

Proof. According to (14), $D^{1}$ writes

$$
\begin{aligned}
D^{1} & =-\int_{\mathbb{R} \times \mathbb{T}} \sigma^{b} \partial_{X}\left(X \partial_{X}{ }^{\alpha} \mathrm{A}\right) \mathrm{d} X \mathrm{~d} Y-\int_{\mathbb{R} \times \mathbb{T}} \sigma^{b} X \partial_{X}^{2}{ }^{\alpha} \mathrm{A} \mathrm{d} X \mathrm{~d} Y \\
& -\varepsilon^{1-\beta} \int_{\mathcal{C}_{\beta ; 1}}\left(\mathfrak{f}_{\varepsilon}\left(M_{\varepsilon}\left[\sigma^{b} \partial_{Y}{ }^{\alpha} \mathrm{A}\right]_{\mathcal{C}_{\beta ; 1}}+n_{\mathcal{C}_{\beta ; 1}}\right)\right) \mathrm{d} s .
\end{aligned}
$$


Then, integrating by parts the first two integrands and using (16) imply that $D^{1}$ equals zero.

For the second property, let $w$ be

$$
\forall X \in \mathbb{R}, \quad w(X)=\left\{\begin{array}{l}
X, \quad \text { if } X>0 \\
\frac{\sigma_{0}}{\sigma_{1}} X, \quad \text { if } X<0,
\end{array}\right.
$$

and define $V$ by

$$
\forall(X, Y) \in \mathbb{R} \times \mathbb{T}, \quad V(X, Y)=\left(\begin{array}{c}
-{ }^{\alpha} \mathrm{A}_{Y}(X, Y) \\
w(X)+{ }^{\alpha} \mathrm{A}_{X}(X, Y)
\end{array}\right)
$$

Observe that according to (14), $V$ satisfies

$$
\begin{aligned}
& \Delta V=0, \text { in } \mathbb{R} \times \mathbb{T} \backslash\left(\mathcal{C}_{0} \cup \mathcal{C}_{\beta ; 1}\right), \\
& \left.\lim _{M \rightarrow+\infty} \partial_{X} V\right|_{M}=\left(\begin{array}{c}
0 \\
1
\end{array}\right), \\
& {\left[\sigma^{b} \partial_{n} V\right]_{\mathcal{C}_{\beta ; 1}}=\left(\sigma_{m}-\sigma_{0}\right) M_{\varepsilon} n_{\mathcal{C}_{0}}, \quad\left[\sigma^{b} \partial_{n} V\right]_{\mathcal{C}_{0}}=0 .}
\end{aligned}
$$

Take $v=V$ in (17), use the expression of $D^{2}$ given by (29) and let $M$ tend to infinity to infer

$$
-\sigma_{0}{ }^{\alpha} \mathrm{a}_{Y}+D_{X}^{2}=\varepsilon^{1-\beta}\left(\int_{0}^{1}\left(V_{X}\left(\varepsilon^{1-\beta} f\left(Y / \varepsilon^{\alpha-\beta}\right), Y\right)-V_{X}(0, Y)\right) \mathrm{d} Y-\int_{0}^{1} V_{Y}\left(\varepsilon^{1-\beta} f\left(Y / \varepsilon^{\alpha-\beta}\right), Y\right) \mathrm{d} Y\right) .
$$

But similar calculations lead to

$$
\begin{aligned}
-\int_{\mathcal{C}_{\beta ; 1}}{ }^{\alpha} \mathrm{A}_{Y}\left[\sigma^{b} \partial_{n}{ }^{\alpha} \mathrm{A}_{X}\right]_{\mathcal{C}_{1 ; \beta}}-\int_{\mathcal{C}_{0}}{ }^{\alpha} \mathrm{A}_{Y}\left[\sigma^{b} \partial_{n}{ }^{\alpha} \mathrm{A}_{X}\right]_{\mathcal{C}_{0}} & =\varepsilon^{1-\beta} \int_{0}^{1}{ }^{\alpha} \mathrm{A}_{X}\left(\varepsilon^{1-\beta} f\left(Y / \varepsilon^{\alpha-\beta}\right), Y\right) \mathrm{d} Y, \\
& =\varepsilon^{1-\beta} \int_{0}^{1} V_{Y}\left(\varepsilon^{1-\beta} f\left(Y / \varepsilon^{\alpha-\beta}\right), Y\right) \mathrm{d} Y
\end{aligned}
$$

and on the other hand, using the jumps (14b)-(14c) we infer

$$
\begin{aligned}
-\int_{\mathcal{C}_{\beta ; 1}}{ }^{\alpha} \mathrm{A}_{Y}\left[\sigma^{b} \partial_{n}{ }^{\alpha} \mathrm{A}_{X}\right]_{\mathcal{C}_{\beta ; 1}}-\int_{\mathcal{C}_{0}}{ }^{\alpha} \mathrm{A}_{Y}\left[\sigma^{b} \partial_{n}{ }^{\alpha} \mathrm{A}_{X}\right]_{\mathcal{C}_{0}} & =-\varepsilon^{1-\beta} \int_{0}^{1}\left({ }^{\alpha} \mathrm{A}_{Y}\left(\varepsilon^{1-\beta} f\left(Y / \varepsilon^{\alpha-\beta}\right), Y\right)\right. \\
& \left.-{ }^{\alpha} \mathrm{A}_{Y}(0, Y)\right) \mathrm{d} Y \\
& =\varepsilon^{1-\beta} \int_{0}^{1}\left(V_{X}\left(\varepsilon^{1-\beta} f\left(Y / \varepsilon^{\alpha-\beta}\right), Y\right)\right. \\
& \left.-V_{X}(0, Y)\right) \mathrm{d} Y
\end{aligned}
$$

hence

$$
D_{X}^{2}=\sigma_{0}^{\alpha} \mathrm{a}_{Y}
$$




\subsection{First order approximation}

It is then possible to obtain the first order coefficient of the expansion. Define $v^{1}$ by

$$
\begin{aligned}
& \Delta_{\eta, \theta} v^{1}=0, \text { in } \mathcal{O}^{1} \cup \mathcal{O}^{0}, \\
& \left.\left(1+d_{0} \kappa\right) \partial_{\eta} v^{1}\right|_{\eta=d_{0}}+\left.\Lambda_{0} v^{1}\right|_{\eta=d_{0}}=0, \\
& \left.\left(1-d_{0} \kappa\right) \partial_{\eta} v^{1}\right|_{\eta=-d_{0}}-\left.\Lambda_{1} v^{1}\right|_{\eta=-d_{0}}=0,
\end{aligned}
$$

with the following transmission conditions:

$$
\begin{aligned}
& \left.\sigma_{0} \partial_{\eta} v^{1}\right|_{\eta=0^{+}}-\left.\sigma_{1} \partial_{\eta} v^{1}\right|_{\eta=0^{-}}=\left.\left(\sigma_{m}-\sigma_{0}\right) D^{2} \cdot \partial_{\theta} \nabla_{\eta, \theta} v\right|_{\eta=0^{+}} ^{0}, \\
& \left.v^{1}\right|_{\eta=0^{+}}-\left.v^{1}\right|_{\eta=0^{-}}=\left.\left(\sigma_{m}-\sigma_{0}\right)^{\alpha} \mathrm{a} \cdot\left(\nabla_{\eta, \theta} v^{0}\right)\right|_{\eta=0^{+}} .
\end{aligned}
$$

Remark 2.4. Observe that the term $\left.{ }^{\alpha} \mathrm{a} \cdot\left(\nabla_{\eta, \theta} v^{0}\right)\right|_{\eta=0^{+}}$is natural, since it comes from the jump of $v_{B L}^{0}$ across the curve $\gamma_{0}$. However the introduction of the slow variable term $D^{2}$, which is obtained by integrating a fast variable term can be seen as artificial. Actually the jump (24) only involves fast variable terms, and we could have thought that they would be corrected in the next boundary layer correctors.

We emphasize that it is necessary to introduce $D^{2}$ so that the next boundary layer correctors be welldefined. Without this term, the next boundary layer correctors would have satisfied ill-posed partial differential equations.

Denote by $w^{1}$ the following quantity:

$$
w^{1}=W^{0}-\varepsilon^{\beta} v^{1} .
$$

Since we have

$$
\left.\partial_{n}^{\Phi} v^{1}\right|_{\gamma_{\varepsilon}}=\left.n_{\mathcal{C}_{1}} \cdot \nabla_{\eta, \theta} v^{1}\right|_{\eta=0^{+}}+O(\varepsilon),
$$

and since $\left.\partial_{\eta} v^{1}\right|_{\eta=0^{+}}=\left.n_{\mathcal{C}_{0}} \cdot \nabla_{\eta, \theta} v^{1}\right|_{\eta=0^{+}}$then $w^{1}$ satisfies:

$$
\begin{aligned}
& \frac{1}{\sigma_{m}-\sigma_{0}} \Delta_{\eta, \theta} w^{1}=\left.\kappa(\theta) G_{1}\left(\eta / \varepsilon^{\beta}, \theta / \varepsilon^{\beta}\right) \cdot \nabla_{\eta, \theta} v^{0}\right|_{\eta=0^{+}}+\left.G_{2}\left(\eta / \varepsilon^{\beta}, \theta / \varepsilon^{\beta}\right) \cdot \partial_{\theta} \nabla_{\eta, \theta} v^{0}\right|_{\eta=0^{+}}+\tilde{O}(\varepsilon), \\
& \left.\left(1+d_{0} \kappa\right) \partial_{\eta} w^{1}\right|_{\eta=d_{0}}+\left.\Lambda_{0} w^{1}\right|_{\eta=d_{0}}=g_{0+}^{\varepsilon}, \\
& \left.\left(1-d_{0} \kappa\right) \partial_{\eta} w^{1}\right|_{\eta=-d_{0}}-\left.\Lambda_{1} w^{1}\right|_{\eta=-d_{0}}=g_{0-}^{\varepsilon},
\end{aligned}
$$

with the transmission conditions:

$$
\begin{aligned}
\frac{1}{\sigma_{m}-\sigma_{0}}\left[\sigma \partial_{n}^{\Phi} w^{1}\right]_{\gamma_{\varepsilon}} & =\varepsilon^{\beta}\left(\left.\kappa(\theta) B_{1}\left(\theta / \varepsilon^{\beta}\right) \cdot \nabla_{\eta, \theta} v^{0}\right|_{\eta=0^{+}}+\left.B_{2}\left(\theta / \varepsilon^{\beta}\right) \cdot \partial_{\theta} \nabla_{\eta, \theta} v^{0}\right|_{\eta=0^{+}}\right) \\
& -\left.\varepsilon^{\beta} n_{\mathcal{C}_{\beta ; 1}} \cdot \nabla_{\eta, \theta} v^{1}\right|_{\eta=0^{+}}+O\left(\varepsilon^{2}\right), \\
\frac{1}{\sigma_{m}-\sigma_{0}}\left[\sigma \partial_{\eta} w^{1}\right]_{\eta=0} & =-\left.\varepsilon^{\beta} D^{2} \cdot \partial_{\theta} \nabla_{\eta, \theta} v^{0}\right|_{\eta=0^{+}}+\left.\varepsilon^{\beta} n_{\mathcal{C}_{0}} \cdot \nabla_{\eta, \theta} v^{1}\right|_{\eta=0^{+}},
\end{aligned}
$$

and

$$
\left.\left[w^{1}\right]\right|_{\gamma_{\varepsilon}}=0,\left.\quad\left[w^{1}\right]\right|_{\eta=0}=0 .
$$


We now introduce two boundary layer correctors at the order 1 defined in $\mathbb{R} \times \mathbb{T}$. For $j=1,2$, the pair $\left({ }^{\alpha} \mathrm{A}^{1, j}, \mathrm{a}^{1, j}\right)$ with ${ }^{\alpha} \mathrm{A}^{1, j}$ continuous, satisfies

$$
\begin{aligned}
& \Delta^{\alpha} \mathrm{A}^{1, j}=G^{j}, \text { in } \mathbb{R} \times \mathbb{T} \backslash\left(\mathcal{C}_{0} \cup \mathcal{C}_{1}\right), \\
& {\left[\sigma^{b} \partial_{n}{ }^{\alpha} \mathrm{A}^{1, j}\right]_{\mathcal{C}_{\beta ; 1}}=B^{j},} \\
& {\left[\sigma^{b} \partial_{n}{ }^{\alpha} \mathrm{A}^{1, j}\right]_{\mathcal{C}_{0}}=-D^{j},} \\
& { }^{\alpha} \mathrm{A}^{1, j} \rightarrow_{X \rightarrow-\infty} 0,{ }^{\alpha} \mathrm{A}^{1, j} \rightarrow_{X \rightarrow+\infty}{ }^{\alpha} \mathrm{a}^{1, j} .
\end{aligned}
$$

According to the definition of $\left(D^{j}\right)_{j=1,2}$, the compatibility condition that ensures both existence and uniqueness of the solution to problem (32),

$$
\int_{\mathbb{R} \times \mathbb{T}} \sigma^{b} G^{j} \mathrm{~d} X \mathrm{~d} Y+\int_{\mathcal{C}_{\beta ; 1}} B^{j} \mathrm{~d} s-\int_{\mathcal{C}_{0}} D^{j} \mathrm{~d} s=0
$$

is satisfied. Define the boundary layer corrector of order $1 v_{B L}^{1}$ on $\mathcal{O}$ by

$$
\begin{aligned}
\forall \eta>0, \quad v_{B L}^{1}(\eta, \theta) & =\left(\sigma_{m}-\sigma_{0}\right) \varepsilon^{2 \beta}\left(\left.\kappa(\theta)\left[{ }^{\alpha} \mathrm{A}^{1,1}\left(\eta / \varepsilon^{\beta}, \theta / \varepsilon^{\beta}\right)-{ }^{\alpha} \mathrm{a}^{1,1}\right] \cdot \nabla_{\eta, \theta} v^{0}\right|_{\eta=0^{+}}\right. \\
& +\left.\left[{ }^{\alpha} \mathrm{A}^{1,2}\left(\eta / \varepsilon^{\beta}, \theta / \varepsilon^{\beta}\right)-{ }^{\alpha} \mathrm{a}{ }^{1,2}\right] \cdot \partial_{\theta} \nabla_{\eta, \theta} v^{0}\right|_{\eta=0^{+}} \\
& \left.+\left.\left[{ }^{\alpha} \mathrm{A}\left(\eta / \varepsilon^{\beta}, \theta / \varepsilon^{\beta}\right)-{ }^{\alpha} \mathrm{a}\right] \cdot \nabla_{\eta, \theta} v^{1}\right|_{\eta=0^{+}}\right), \\
\forall \eta<0, \quad v_{B L}^{1}(\eta, \theta) & =\left(\sigma_{m}-\sigma_{0}\right) \varepsilon^{2 \beta}\left(\left.\kappa(\theta)^{\alpha} \mathrm{A}^{1,1}\left(\eta / \varepsilon^{\beta}, \theta / \varepsilon^{\beta}\right) \cdot \nabla_{\eta, \theta} v^{0}\right|_{\eta=0^{+}}\right. \\
& \left.+\left.{ }^{\alpha} \mathrm{A}^{1,2}\left(\eta / \varepsilon^{\beta}, \theta / \varepsilon^{\beta}\right) \cdot \partial_{\theta} \nabla_{\eta, \theta} v^{0}\right|_{\eta=0^{+}}+\left.{ }^{\alpha} \mathrm{A}\left(\eta / \varepsilon^{\beta}, \theta / \varepsilon^{\beta}\right) \cdot \nabla_{\eta, \theta} v^{1}\right|_{\eta=0^{+}}\right) .
\end{aligned}
$$

\section{ERror estimates FOR $\alpha \in(0,2)$}

In the previous section we have formally derived the first order of the asymptotic expansion of $v^{\varepsilon}$, by defining appropriate boundary layer correctors. The aim of the section is to prove that this formal construction is indeed an approximation of $v^{\varepsilon}$ at the first order.

Theorem 3.1. Let $\alpha \in(0,2)$. Suppose the boundary data g be smooth ${ }^{3}$. Define $W^{1}$ by

$$
W^{1}=v^{\varepsilon}-\left(v^{0}+v_{B L}^{0}+\varepsilon^{\beta}\left(v^{1}+v_{B L}^{1}\right)\right) .
$$

Then, $W^{1}$ is $H^{1}-$ regular in each subdomain $\mathcal{O}_{0}$ and $\mathcal{O}_{1}$, and it satisfies

$$
\left\|W^{1}\right\|_{H^{1}\left(\mathcal{O}_{0}\right)}+\left\|W^{1}\right\|_{H^{1}\left(\mathcal{O}_{1}\right)}=o(\varepsilon) .
$$

Remark 3.2. The above theorem ensures that $v^{0}+v_{B L}^{0}+\varepsilon^{\beta}\left(v^{1}+v_{B L}^{1}\right)$ gives an approximation of $v^{\varepsilon}$ at the order $o(\varepsilon)$ in the whole domain $\Omega$. In particular, we have provided an accurate description of $v^{\varepsilon}$ in a neighborhood of the rough thin layer, which was not obtained by the analysis derived by Vogelius et al.

\footnotetext{
${ }^{3}$ This hypothesis can be weakened to $g \in H^{s}(\partial \Omega)$ for an index $s$ large enough.
} 
For $\alpha \leq 1$, more accurate estimates hold:

$$
\left\|W^{1}\right\|_{H^{1}\left(\mathcal{O}_{0}\right)}+\left\|W^{1}\right\|_{H^{1}\left(\mathcal{O}_{1}\right)}=O\left(\varepsilon^{2}\right) .
$$

Proof. According to the derivation of the first order terms, the function $W^{1}$ satisfies the following boundary value problem:

$$
\begin{aligned}
& \Delta_{\eta, \theta} W^{1}=F_{\varepsilon}, \text { in } \mathcal{O}^{1} \cup \mathcal{O}_{\varepsilon}^{m} \cup \mathcal{O}_{\varepsilon}^{0}, \\
& \left.\left(1+d_{0} \kappa\right) \partial_{\eta} W^{1}\right|_{\eta=d_{0}}+\left.\Lambda_{0} W^{1}\right|_{\eta=d_{0}}=g_{1+}^{\varepsilon}, \\
& \left.\left(1-d_{0} \kappa\right) \partial_{\eta} W^{1}\right|_{\eta=-d_{0}}-\left.\Lambda_{1} W^{1}\right|_{\eta=-d_{0}}=g_{1-}^{\varepsilon},
\end{aligned}
$$

with the following transmission conditions:

$$
\begin{aligned}
& \left.\sigma_{0} \partial_{n}^{\Phi} W^{1}\right|_{\gamma_{\varepsilon}^{+}}=\left.\sigma_{m} \partial_{n}^{\Phi} W^{1}\right|_{\gamma_{\varepsilon}^{-}}+\varepsilon^{1+\beta} R_{1}^{\varepsilon}, \\
& \left.\sigma_{m} \partial_{\eta} W^{1}\right|_{\eta=0^{+}}=\left.\sigma_{1} \partial_{\eta} W^{1}\right|_{\eta=0^{-}}+\varepsilon^{1+\beta} R_{2}^{\varepsilon},
\end{aligned}
$$

and

$$
\begin{aligned}
& \left.W^{1}\right|_{\gamma_{\varepsilon}^{+}}=\left.W^{1}\right|_{\gamma_{\varepsilon}^{-}}, \\
& \left.W^{1}\right|_{\eta=0^{+}}=\left.W^{1}\right|_{\eta=0^{-}}+\varepsilon^{1+\beta} R_{3}^{\varepsilon} .
\end{aligned}
$$

Let $\mathfrak{L}\left(\gamma_{\varepsilon}\right)$ be the arc length of $\gamma_{\varepsilon}$, which equals

$$
\mathfrak{L}\left(\gamma_{\varepsilon}\right)=\sqrt{1+\varepsilon^{1-\alpha} f^{\prime}\left(\theta / \varepsilon^{\alpha}\right)} .
$$

For $\alpha \leq 1$, since $\mathfrak{L}\left(\gamma_{\varepsilon}\right)$ is bounded with respect to $\varepsilon$, therefore for any smooth function $\varphi$ defined in $\mathcal{O}$, the following equality holds

$$
\left|\varphi_{\gamma_{\varepsilon}}\right|_{L^{2}\left(\gamma_{\varepsilon}\right)}=\int_{0}^{1}\left|\varphi\left(\varepsilon f\left(\theta / \varepsilon^{\alpha}, \theta\right)\right)\right|^{2} \mathfrak{L}\left(\gamma_{\varepsilon}\right) \mathrm{d} \theta
$$

ensuring the boundedness of $\left|\varphi_{\left.\right|_{\varepsilon}}\right|_{L^{2}\left(\gamma_{\varepsilon}\right)}$ by $\|\varphi\|_{H^{1}(\mathcal{O})}$ uniformly with respect to $\varepsilon$. Observe that according to estimates (15), the source terms $R_{j}^{\varepsilon}$, for $j=1,2$ are of order $\varepsilon^{1-\beta}$, hence following the proof of Theorem 4.1 of Ciuperca et al., we infer that

$$
\left\|W^{1}\right\|_{H^{1}\left(\mathcal{O}_{0}\right)}+\left\|W^{1}\right\|_{H^{1}\left(\mathcal{O}_{1}\right)}=O\left(\varepsilon^{2}\right) .
$$

For $\alpha \in(1,2)$ the arc length $\mathfrak{L}\left(\gamma_{\varepsilon}\right)$ blows up like $\varepsilon^{1-\alpha}$ hence

$$
\left\|\varphi_{\left.\right|_{\gamma_{\varepsilon}}}\right\|_{L^{2}\left(\gamma_{\varepsilon}\right)} \leq C \varepsilon^{1-\alpha}\|\varphi\|_{H^{1}(\mathcal{O})} .
$$

Moreover, since $\beta$ equals 1 in this case, estimates (15) do not provide any gain in power of $\varepsilon$, hence

$$
\left\|W^{1}\right\|_{H^{1}\left(\mathcal{O}_{0}\right)}+\left\|W^{1}\right\|_{H^{1}\left(\mathcal{O}_{1}\right)}=O\left(\varepsilon^{2+\beta-\alpha}\right)=o(\varepsilon),
$$

since $\alpha<2$.

Remark 3.3. As soon as $\alpha \geq 2$, the estimate dramatically crashes since the arc length $\mathfrak{L}\left(\gamma_{\varepsilon}\right)$ cannot be compensated by the first order term of the asymptotic. It is therefore necessary to derive higher order terms in the expansion of $v^{\varepsilon}$ in order to obtain appropriate results. This is quite technical, but we are confident that the reader has all the tools to push forward the expansion at the desired order. 


\subsection{Generalized Polarization Tensor}

Generalized polarization tensor as described by Vogelius, Ammari, Kang et al. $[4,5,7,9]$ has a wide range of applications, in particular in the inverse problem research area (see the book of Ammari and Kang [5] and references therein). In this section we provide an explicit characterization of the polarization tensor using the zeroth order boundary layer corrector. Denote by $G(x, y)$ the Dirichlet solution for the Laplace operator defined in $[4,5]$ p. 33 by

$$
\left\{\begin{array}{l}
\nabla_{x} \cdot\left(\sigma \nabla_{x} G(x, y)\right)=-\delta_{y}, \text { in } \Omega, \\
G(x, y)=0, \quad \forall x \in \partial \Omega .
\end{array}\right.
$$

Let $K$ be a tubular neighborhood of $\partial \mathcal{O}^{1}$ and let $\varepsilon_{0}$ be such that for any $\varepsilon \in(0, \varepsilon), \mathcal{O}_{\varepsilon}^{m} \subset K$. According to the definition of $v^{1}$ and to theorem 3.1, the following equality holds almost everywhere far from the layer:

$$
v^{\varepsilon}(y)-v^{0}(y)=\varepsilon \int_{\Gamma}\left(\sigma_{m}-\sigma_{0}\right) \mathcal{M}_{\alpha}\left(\begin{array}{c}
\partial_{n} u \\
\nabla_{\Gamma} u
\end{array}\right) \cdot\left(\begin{array}{c}
\partial_{n} G \\
\nabla_{\Gamma} G
\end{array}\right)(s, y) \mathrm{d}_{\Gamma}(s)+o(\varepsilon), \quad \text { a.e. } \mathcal{O} \backslash K,
$$

where $\mathcal{M}_{\alpha}$ is the polarization tensor defined by

$$
\mathcal{M}_{\alpha}=\varepsilon^{\beta-1}\left(\begin{array}{cc}
\sigma_{0}^{\alpha} \mathrm{a}_{X} & \sigma_{0}{ }^{\alpha} \mathrm{a}_{Y} \\
D_{X}^{2} & D_{Y}^{2}
\end{array}\right) .
$$

According to estimates (15) and using the definition (29) of $D^{2}$, the polarization tensor is of order $O(1)$. Property 2.3 ensures the symmetry of $M$, as proved in [9]. It is also worth noting that the polarization tensor does not depend on the curvature of the domain, but only on the characteristics of the oscillations, since the boundary layer corrector $\left({ }^{\alpha} \mathrm{A},{ }^{\alpha} \mathrm{a}\right)$ does not depend on the curvature. Therefore, $\left({ }^{\alpha} \mathrm{A},{ }^{\alpha} \mathrm{a}\right)$ leads to define explicitly the polarization tensor for rough thin layer, for any roughness parameter $\alpha \in(0,2)$.

In addition, an accurate approximation at the order $o(\varepsilon)$ is obtained in a neighborhood of the rough thin layer, whereas the variational techniques used by Vogelius et al. provide estimates that are valid far from the layer.

We thus have provided a link between boundary layer correctors and polarization tensor for rough thin layers, for a roughness parameter $\alpha \in(0,2)$. Observe that as soon as $\alpha<1$, the corrector $\left({ }^{\alpha} \mathrm{A},{ }^{\alpha} \mathrm{a}\right)$ depends on $\varepsilon^{1-\alpha}$, while for $\alpha>1$ it depends on $\varepsilon^{\alpha-1}$ : in the next section we explicitly characterize the leading term of $\left({ }^{\alpha} \mathrm{A},{ }^{\alpha} \mathrm{a}\right)$ for $\alpha \neq 1$.

\section{Leading term of the Corrector $\left({ }^{\alpha} \mathrm{A},{ }^{\alpha} \mathrm{a}\right)$ For $\beta \neq 1$}

In the previous section we have derived the first order boundary layer correctors $\left({ }^{\alpha} \mathrm{A},{ }^{\alpha} \mathrm{a}\right)$, involving the parameter $\beta$, which is nothing but the minimum between $\alpha$ and 1 . For $\alpha=1$ the problem satisfied by $\left({ }^{\alpha} \mathrm{A},{ }_{\mathrm{a}}\right)$ does not involve any small parameter, but as soon as $\alpha \neq 1$ it does. The aim of this section is to provide the leading term of $\left({ }^{\alpha} \mathrm{A},{ }^{\alpha} \mathrm{a}\right)$ in the two different cases $\alpha<1$ and $\alpha>1$.

\subsection{The weakly oscillating case: $\alpha \in(0,1)$}

According to property $2.1,{ }^{\alpha} \mathrm{A}$ is bounded by $\varepsilon^{1-\beta}$. Therefore, for $\alpha<1$, it is possible to approximate $\left({ }^{\alpha} \mathrm{A},{ }^{\alpha} \mathrm{a}\right)$ by its leading term. This case is quite simple, since it deals with a smooth thin layer of non-constant thickness and has been previously described in [16]. More precisely, the geometry is described by figure 6 .

Performing the rescaling $\Xi=X / \varepsilon^{1-\beta}$ in $Y_{m}^{\beta}$, and denoting by ${ }^{\alpha} V$ the profile:

$$
\forall(\Xi, Y) \in\{Y \in \mathbb{T}, 0<\Xi<f(Y)\}, \quad{ }^{\alpha} V(\Xi, Y)={ }^{\alpha} \mathrm{A}\left(\varepsilon^{1-\beta} \Xi, Y\right),
$$




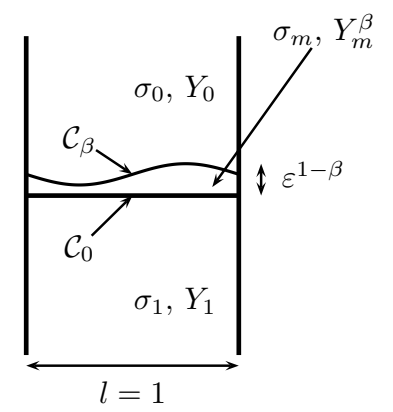

Figure 6. Geometry of the strip

we infer

$$
\begin{aligned}
& \Delta_{X, Y}{ }^{\alpha} \mathrm{A}=0, \text { in }\{X<0\} \cup\left\{X>\varepsilon^{1-\beta} f(Y)\right\}, \\
& \partial_{\Xi}^{2} V+\left(\varepsilon^{1-\beta}\right)^{2} \partial_{Y}{ }^{\alpha} V=0, \text { in }\{Y \in \mathbb{T}, 0<\Xi<f(Y)\}, \\
& \left.\sigma_{0} \nabla_{X, Y}{ }^{\alpha} \mathrm{A}\right|_{\left(\varepsilon^{1-\beta} f(Y), Y\right)} \cdot n_{\mathcal{C}_{\beta ; 1}}-\left.\sigma_{m}\left(\begin{array}{c}
\frac{1}{\varepsilon^{1-\beta}} \partial_{\Xi}{ }^{\alpha} V \\
\partial_{Y}{ }^{\alpha} V
\end{array}\right)\right|_{\Xi=f(Y)} \cdot n_{\mathcal{C}_{\beta ; 1}}=n_{\mathcal{C}_{\beta ; 1}}, \\
& \left.\sigma_{1} \partial_{X}{ }^{\alpha} \mathrm{A}\right|_{0, Y}-\left.\sigma_{m} \frac{1}{\varepsilon^{1-\beta}} \partial_{\Xi}{ }^{\alpha} V\right|_{(0, Y)}=-n_{\mathcal{C}_{0}}, \\
& \left.\left.{ }^{\alpha} \mathrm{A}\right|_{0, Y}=\left.{ }^{\alpha} V\right|_{(0, Y)},\left.\quad{ }^{\alpha} \mathrm{A}\right|_{\left(\varepsilon^{1-\beta}\right.} f(Y), Y\right) \\
& \left.{ }^{\alpha} V\right|_{(f(Y), Y)}, \\
& { }^{\alpha} \mathrm{A} \rightarrow X \rightarrow-\infty \\
&
\end{aligned}
$$

Then, assuming the ansatz

$$
\begin{aligned}
& { }^{\alpha} \mathrm{A}=\sum_{n \geq 0}\left(\varepsilon^{1-\beta}\right)^{n} A_{n}, \\
& { }^{\alpha} V=\sum_{n \geq 0}\left(\varepsilon^{1-\beta}\right)^{n} V_{n},
\end{aligned}
$$

and identifying the terms with the same power in $\varepsilon^{1-\beta}$ we infer that $A_{0}$ identically equals 0 , and that $A_{1}$ equals

$$
A_{1}=\left\{\begin{array}{l}
0, \quad \text { if } X<0, \\
\frac{1}{\sigma_{m}}\left(\begin{array}{l}
f \\
0
\end{array}\right), \quad \text { if } X>0,
\end{array}\right.
$$

from which we infer that

$$
\alpha_{\mathrm{a}}=\varepsilon^{1-\beta} \frac{1}{\sigma_{m}}\left(\begin{array}{c}
\int_{\mathbb{T}} f(Y) \mathrm{d} Y \\
0
\end{array}\right)+O\left(\varepsilon^{1-\beta}\right)
$$

and

$$
D^{2}=\varepsilon^{1-\beta}\left(\begin{array}{c}
0 \\
\int_{\mathbb{T}} f(Y) \mathrm{d} Y
\end{array}\right)+O\left(\varepsilon^{1-\beta}\right) .
$$


Therefore, setting $\tilde{v}^{1}=v^{1} / \varepsilon^{1-\beta}$, we infer

$$
\begin{aligned}
& \Delta_{\eta, \theta} \tilde{v}^{1}=0, \text { in } \mathcal{O}^{1} \cup \mathcal{O}^{0}, \\
& \left.\left(1+d_{0} \kappa\right) \partial_{\eta} \tilde{v}^{1}\right|_{\eta=d_{0}}+\left.\Lambda_{0} \tilde{v}^{1}\right|_{\eta=d_{0}}=0, \\
& \left.\left(1-d_{0} \kappa\right) \partial_{\eta} \tilde{v}^{1}\right|_{\eta=-d_{0}}-\left.\Lambda_{1} \tilde{v}^{1}\right|_{\eta=-d_{0}}=0,
\end{aligned}
$$

with the following transmission conditions:

$$
\begin{aligned}
& \left.\sigma_{0} \partial_{\eta} \tilde{v}^{1}\right|_{\eta=0^{+}}-\left.\sigma_{1} \partial_{\eta} \tilde{v}^{1}\right|_{\eta=0^{-}}=\left.\left(\sigma_{m}-\sigma_{0}\right) \bar{f} \partial_{\theta}^{2} v\right|_{\eta=0^{+}} ^{0}, \\
& \left.\tilde{v}^{1}\right|_{\eta=0^{+}}-\left.\tilde{v}^{1}\right|_{\eta=0^{-}}=\left.\frac{\sigma_{m}-\sigma_{0}}{\sigma_{m}} \bar{f} \partial_{\eta} v^{0}\right|_{\eta=0^{+}},
\end{aligned}
$$

where

$$
\bar{f}=\int_{\mathbb{T}} f(Y) \mathrm{d} Y .
$$

According to theorem 3.1, we have shown that far from the layer:

$$
v^{\varepsilon}-v^{0}=\varepsilon \tilde{v}^{1}+o(\varepsilon)
$$

which means that the mean magnitude of the roughness provides a first order approximation of $v^{\varepsilon}$, as previously described in [16], and the polarization tensor writes:

$$
\mathcal{M}_{\alpha}=\bar{f}\left(\begin{array}{cc}
\frac{\sigma_{0}}{\sigma_{m}} & 0 \\
0 & 1
\end{array}\right)
$$

as obtained by Beretta et al. $[7,8]$ in the case of constant thickness $(\alpha=0)$.

Observe that as $\beta \in(0,1)$ goes to 1 the convergence rate of $\varepsilon^{1-\beta} A_{1}$ to ${ }^{\alpha} \mathrm{A}$ decreases, meaning that the mean-value of the roughness is no more sufficient to approach $v^{\varepsilon}$ : the roughness effect becomes as important as the layer thickness, and the tangential component of the zeroth order potential $v^{0}$ is necessary to define $v^{1}$.

\subsection{The very oscillating case $\alpha>1$ : two-scale limit of the boundary layer}

We now focus on the case $\alpha>1$. Then, $\varepsilon^{\alpha-1}$ goes to zero, and we denote by $\delta$ this small parameter. Denote by $\mathcal{C}_{0}$ and $\mathcal{C}_{\delta}$ the closed curves defined by

$$
\mathcal{C}_{0}=\{0\} \times \mathbb{T}, \quad \mathcal{C}_{\delta}=\{(f(Y / \delta), Y), \forall Y \in \mathbb{T}\}, \quad \text { with } \delta=\varepsilon^{\alpha-1} .
$$

The exterior normal to $\mathcal{C}_{\delta}$ is denoted by $n_{\mathcal{C}_{\delta}}$, and $n_{\mathcal{C}_{0}}$ is the exterior normal to $\mathcal{C}_{0}$ :

$$
n_{\mathcal{C}_{0}}=\left(\begin{array}{l}
1 \\
0
\end{array}\right), \quad n_{\mathcal{C}_{\delta}}=\frac{1}{\sqrt{1+\left(f^{\prime}(Y / \delta) / \delta\right)^{2}}}\left(\begin{array}{c}
1 \\
-f^{\prime}(Y / \delta) / \delta
\end{array}\right) .
$$

Let $\sigma^{\sharp}$ be the function defined by

$$
\forall(X, \tau) \in \mathbb{R} \times \mathbb{T}, \quad \sigma^{\sharp}(X, \tau)= \begin{cases}\sigma_{0}, & \text { in }\{(X, \tau), \quad \tau \in \mathbb{T}, X>f(\tau)\}, \\ \sigma_{m}, & \text { in }\{(X, \tau), \quad \tau \in \mathbb{T}, 0<x<f(\tau)\}, \\ \sigma_{1}, & \text { in }\{(X, \tau), \quad \tau \in \mathbb{T}, X<0\} .\end{cases}
$$


Observe that

$$
\forall(X, Y) \in \mathbb{R} \times \mathbb{T}, \quad \sigma^{b}(X, Y)=\sigma^{\sharp}(X, Y / \delta) .
$$

To highlight the dependence on $\delta$ we rewrite $\sigma^{b}$ as $\sigma_{\delta}^{b}$. Since we focus on the case $\beta=1$, we rewrite $\left({ }^{\alpha} \mathrm{A},{ }^{\alpha} \mathrm{a}\right)$

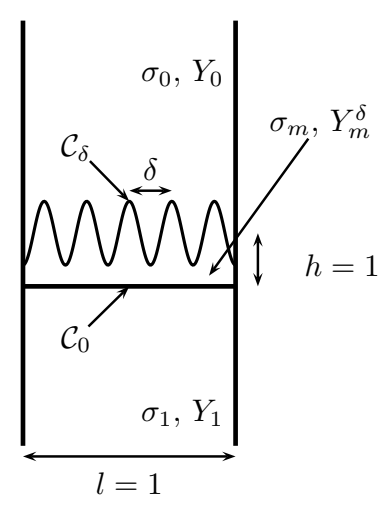

FiguRe 7. Rescaled strip

in $\left(\mathfrak{A}^{\delta}, \mathfrak{a}^{\delta}\right)$. We recall that $\mathfrak{A}^{\delta}$ is a continuous vector field and $\mathfrak{a}^{\delta}$ is a constant vector, which are given by the unique solution to the following problem

$$
\begin{aligned}
& \Delta \mathfrak{A}^{\delta}=0, \text { in } \mathbb{R} \times \mathbb{T} \backslash\left(\mathcal{C}_{0} \cup \mathcal{C}_{\delta}\right), \\
& {\left[\sigma_{\delta}^{b} \partial_{n} \mathfrak{A}^{\delta}\right]_{\mathcal{C}_{\delta}}=n_{\mathcal{C}_{\delta}},} \\
& {\left[\sigma_{\delta}^{b} \partial_{n} \mathfrak{A}^{\delta}\right]_{\mathcal{C}_{0}}=-n_{\mathcal{C}_{0}},} \\
& \mathfrak{A}^{\delta} \rightarrow_{x \rightarrow-\infty} 0, \mathfrak{A}^{\delta} \rightarrow_{x \rightarrow+\infty} \mathfrak{a}^{\delta} .
\end{aligned}
$$

Remark 4.1. For all $\delta>0$, the existence and the uniqueness of the pair $\left(\mathfrak{A}^{\delta}, \mathfrak{a}^{\delta}\right)$ are shown in property 2.1 . We recall that the convergence rates are exponential as proved in [11]. We aim at deriving the limit corrector as $\delta$ goes to zero.

Denote by $\mathscr{E}^{\sharp}$ be

$$
\mathscr{E}^{\sharp}=\left\{\begin{array}{c}
\phi \in\left(H_{l o c}^{1}(\mathbb{R} \times \mathbb{T} \times \mathbb{T})\right)^{2}: \phi \text { is } Y \text {-periodic and } \tau \text {-periodic, } \\
\int_{\mathbb{R} \times \mathbb{T} \times \mathbb{T}}\|\nabla \phi(X, Y, \tau)\|^{2} \mathrm{~d} X \mathrm{~d} Y \mathrm{~d} \tau<+\infty ; \quad \phi \rightarrow_{X \rightarrow-\infty} 0
\end{array}\right\} .
$$

Observe that the variational formulation of problem (35) is

$$
\left\{\begin{array}{l}
\text { Find } \mathfrak{A}^{\delta} \in \mathscr{E} \text { such that for all } \phi \in \mathscr{E} \\
\int_{\mathbb{R} \times \mathbb{T}} \sigma_{\delta}^{b} \nabla \mathfrak{A}^{\delta} \cdot \nabla \phi \mathrm{d} X \mathrm{~d} Y=\int_{0}^{1} \int_{0}^{f(Y / \delta)} \nabla \cdot \phi(X, Y) \mathrm{d} X \mathrm{~d} Y .
\end{array}\right.
$$


We remind that a straightforward application of Lax-Milgram lemma leads to existence and uniqueness of $\mathfrak{A}^{\delta}$, which were obtained previously in [11]. Moreover, from the variational formulation (36) we derive straightforwardly the following estimate as

$$
\int_{\mathbb{R} \times \mathbb{T}}\left\|\nabla \mathfrak{A}^{\delta}(X, Y)\right\|^{2} \mathrm{~d} X \mathrm{~d} Y \leq \frac{1}{\min \left(\sigma_{0}, \sigma_{m}, \sigma_{1}\right)} .
$$

Therefore, there exists a $2 \times 2$ matrix-function $\chi^{0}$ defined on $\mathbb{R} \times \mathbb{T} \times \mathbb{T}$ such that $\chi^{0}(\cdot, \cdot, \tau)$ belongs to $\mathscr{E}$ for almost all $\tau \in \mathbb{T}$ and, up to a subsequence, $\nabla \mathfrak{A}^{\delta}$ two-scale converges to $\chi^{0}$.

Let $\left(\varphi^{0}, \varphi^{1}\right)$ belong to $\mathscr{E} \times \mathscr{E}^{\sharp}$. We rewrite equality (36) with

$$
\phi(X, Y)=\varphi^{0}(X, Y)+\delta \varphi^{1}(X, Y, Y / \delta) .
$$

Passing to the limit for $\delta$ tending to zero implies

$$
\int_{\mathbb{R} \times \mathbb{T} \times \mathbb{T}} \sigma^{\sharp} \chi^{0} \cdot\left(\nabla_{X, Y} \varphi^{0}+\nabla_{\tau} \varphi^{1}\right) \mathrm{d} X \mathrm{~d} Y \mathrm{~d} \tau=\int_{\mathbb{T} \times \mathbb{T}} \int_{0}^{f(\tau)}\left(\nabla_{X, Y} \cdot \varphi^{0}+\partial_{\tau} \varphi_{Y}^{1}\right) \mathrm{d} X \mathrm{~d} Y \mathrm{~d} \tau,
$$

where for $\psi \in \mathscr{E}^{\sharp \sharp}, \nabla_{\tau} \psi$ denotes the following matrix

$$
\nabla_{\tau} \psi=\left(\begin{array}{cc}
0 & \partial_{\tau} \psi_{X} \\
0 & \partial_{\tau} \psi_{Y}
\end{array}\right)
$$

Suppose that $\chi^{0}$ writes

$$
\forall(X, Y, \tau) \in \mathbb{R} \times \mathbb{T} \times \mathbb{T}, \quad \chi^{0}(X, Y, \tau)=\nabla_{X, Y} \mathfrak{A}^{0}(X, Y)+\nabla_{\tau} \mathfrak{A}^{1}(X, Y, \tau) .
$$

\subsubsection{Problem satisfied by $\mathfrak{A}^{1}$}

Denote by $q$ the cumulative distribution function defined by

$$
\forall X \in \mathbb{R}, \quad q(X)=\int_{\mathbb{T}} \mathbb{1}_{\{0<X<f(\tau)\}} \mathrm{d} \tau .
$$

From equality (38), by taking $\varphi^{0}$ identically equal to zero, we infer that for any $\psi \in \mathscr{E}^{\sharp}$

$$
\int_{\mathbb{R} \times \mathbb{T} \times \mathbb{T}} \sigma^{\sharp}(X, \tau) \partial_{\tau} \mathfrak{A}^{1} \cdot \partial_{\tau} \psi \mathrm{d} X \mathrm{~d} Y \mathrm{~d} \tau=\int_{\mathbb{R} \times \mathbb{T} \times \mathbb{T}}\left(\begin{array}{c}
-\sigma^{\sharp}(X, \tau) \partial_{Y} \mathfrak{A}_{X}^{0} \\
-\sigma^{\sharp}(X, \tau) \partial_{Y} \mathfrak{A}_{Y}^{0}+\mathbb{1}_{\{0<X<f(\tau)\}}
\end{array}\right) \cdot \partial_{\tau} \psi \mathrm{d} X \mathrm{~d} Y \mathrm{~d} \tau,
$$

hence the ordinary differential equation satisfied by $\mathfrak{A}^{1}$ :

$$
\partial_{\tau}\left(\sigma^{\sharp} \partial_{\tau} \mathfrak{A}^{1}\right)=\frac{\partial}{\partial \tau}\left(\begin{array}{c}
-\sigma^{\sharp} \partial_{Y} \mathfrak{A}_{X}^{0} \\
-\sigma^{\sharp} \partial_{Y} \mathfrak{A}_{Y}^{0}+\mathbb{1}_{\{0<X<f(\tau)\}}
\end{array}\right) .
$$

Since $\int_{\mathbb{T}} \partial_{\tau} \mathfrak{A}^{1} \mathrm{~d} \tau=0$ we deduce

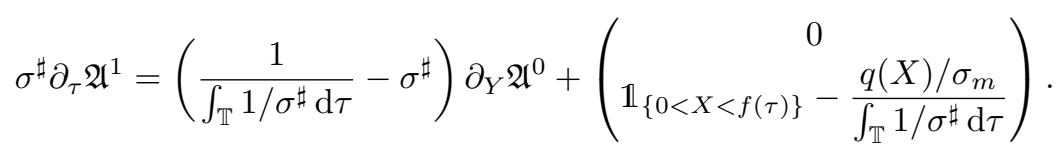

Observe that

$$
\forall X<0, \forall(Y, \tau) \in \mathbb{T} \times \mathbb{T}, \quad \sigma^{\sharp} \partial_{\tau} \mathfrak{A}^{1}(X, Y, \tau)=0 .
$$




\subsubsection{Problem satisfied by $\mathfrak{A}^{0}$}

From equality (38), by taking $\varphi^{1}$ independent of $\tau$, we infer

$$
\int_{\mathbb{R} \times \mathbb{T}}\left(\int_{\mathbb{T}} \sigma^{\sharp}(X, \tau)\left(\nabla_{X, Y} \mathfrak{A}^{0}(X, Y)+\nabla_{\tau} \mathfrak{A}^{1}(X, Y, \tau)\right) \mathrm{d} \tau\right) \cdot \nabla_{X, Y} \varphi^{0} \mathrm{~d} X \mathrm{~d} Y=\int_{\mathbb{R} \times \mathbb{T}} q(X) \partial_{X} \varphi_{X}^{0} \mathrm{~d} X \mathrm{~d} Y .
$$

Hence, denoting by $\bar{\sigma}^{\sharp}$ the function

$$
\forall X \in \mathbb{R}, \quad \bar{\sigma}^{\sharp}(X)=\int_{\mathbb{T}} \sigma^{\sharp}(X, \tau) \mathrm{d} \tau,
$$

we infer that $\mathfrak{A}^{0}$ satisfies

$$
\begin{aligned}
& \nabla \cdot\left(\bar{\sigma}^{\sharp} \nabla \mathfrak{A}^{0}\right)=\nabla_{X, Y} q-\nabla_{X, Y} \cdot\left(\int_{\mathbb{T}} \sigma^{\sharp}(., \tau) \nabla_{\tau} \mathfrak{A}^{1}(., ., \tau) \mathrm{d} \tau\right), \quad \text { in }\{X>0\} \times \mathbb{T}, \\
& \Delta \mathfrak{A}^{0}=0, \quad \text { in }\{X<0\} \times \mathbb{T}, \\
& {\left[\bar{\sigma}^{\sharp} \partial_{n} \mathfrak{A}^{0}\right]_{\mathcal{C}_{0}}=n_{\mathcal{C}_{0}} .}
\end{aligned}
$$

Therefore, according to (39)-(41), we infer the strong formulation for $\mathfrak{A}^{0}$, which is continuous and satisfies:

$$
\begin{aligned}
& \partial_{X}\left(\bar{\sigma}^{\sharp} \partial_{X} \mathfrak{A}^{0}\right)+\frac{1}{\int_{\mathbb{T}} 1 / \sigma^{\sharp} \mathrm{d} \tau} \partial_{Y}^{2} \mathfrak{A}^{0}=\nabla q, \quad \text { in }\{X>0\} \times \mathbb{T}, \\
& \Delta \mathfrak{A}^{0}=0, \quad \text { in }\{X<0\} \times \mathbb{T}, \\
& {\left[\bar{\sigma}^{\sharp} \partial_{X} \mathfrak{A}^{0}\right]_{\mathcal{C}_{0}}=n_{\mathcal{C}_{0}},} \\
& \mathfrak{A}^{0} \rightarrow X \rightarrow-\infty 0, \quad \mathfrak{A}^{0} \rightarrow X \rightarrow+\infty \mathfrak{a}^{0} .
\end{aligned}
$$

Remark 4.2. We recall that

$$
n_{\mathcal{C}_{0}}=\left(\begin{array}{l}
1 \\
0
\end{array}\right), \text { and } \quad\left[\bar{\sigma}^{\sharp} \partial_{n} \mathfrak{A}^{0}\right]_{\mathcal{C}_{0}}=\left.\sigma_{m} \partial_{X} \mathfrak{A}^{0}\right|_{X=0^{+}}-\left.\sigma_{1} \partial_{X} \mathfrak{A}^{0}\right|_{X=0^{-}}
$$

We emphasize that since $q(0)=1$ and $q(M)=0$ for all $M>\|f\|_{\infty}$, the compatibility condition to ensure existence and uniqueness of $\mathfrak{A}^{0}$ is satisfied (see [11] Lemma 2.2).

Equation (42a) can be rewritten as

$$
\nabla \cdot\left(\left(\begin{array}{cc}
\bar{\sigma}^{\sharp} & 0 \\
0 & 1 /\left(\int_{\mathbb{T}}^{\sharp} \sigma^{\sharp} \mathrm{d} \tau\right)
\end{array}\right) \nabla \mathfrak{A}^{0}\right)=\nabla q,
$$

and this has to be related with the well-known homogenization formula for laminate structures: in the laminate alignment (here the $X$-direction), the mean of the conductivities appears, while in the transverse direction (the $Y$-direction in our case) the harmonic mean holds.

4.2.3. Computation of the coefficients $\mathfrak{a}^{0}$ and $D^{2}$.

Observe that the second component of $\mathfrak{a}^{0}$ is necessarily equal to 0 , since all the source terms vanish according to (42a). To obtain the value of the first component $\mathfrak{a}_{X}^{0}$, multiply (42a) by a test function $v \in H^{2}([-M, M] \times \mathbb{T})$, 
which is independent of $Y$ and integrate by parts twice to obtain

$$
\begin{aligned}
& \left.\sigma_{0} \int_{0}^{1} \mathfrak{A}_{X}^{0}(M, Y) \partial_{X} v\right|_{M} \mathrm{~d} Y-\left.\sigma_{1} \int_{0}^{1} \mathfrak{A}_{X}^{0}(-M, Y) \partial_{X} v\right|_{-M} \mathrm{~d} Y-\left.\sigma_{0} \int_{0}^{1} \partial_{X} \mathfrak{A}_{X}^{0}(M, y) v\right|_{M} \mathrm{~d} Y, \\
& +\left.\sigma_{1} \int_{0}^{1} \partial_{X} \mathfrak{A}_{X}^{0}(-M, y) v\right|_{-M} \mathrm{~d} Y-\int_{(-M, 0) \times \mathbb{T}} \sigma_{1} \mathfrak{A}_{X}^{0} \cdot \partial_{X}^{2} v \mathrm{~d} X \mathrm{~d} Y-\int_{(0, M) \times \mathbb{T}} \mathfrak{A}_{X}^{0} \cdot \partial_{X}\left(\bar{\sigma}^{\sharp} \partial_{X} v\right) \mathrm{d} X \mathrm{~d} Y \\
& =-\int_{0}^{+\infty} \int_{\mathbb{T}} q(X) \partial_{X} v(X) \mathrm{d} X .
\end{aligned}
$$

Now choose $v$ as follows:

$$
v(X)=\left\{\begin{array}{l}
\int_{0}^{X} 1 / \bar{\sigma}^{\sharp}(s) \mathrm{d} s, \quad \text { if } X>0, \\
X / \sigma_{1}, \quad \text { if } X<0,
\end{array}\right.
$$

to infer the value of $\mathfrak{a}_{X}^{0}$ :

$$
\mathfrak{a}_{X}^{0}=-\int_{0}^{+\infty} \frac{q(s)}{\bar{\sigma}^{\sharp}(s)} \mathrm{d} s .
$$

According to property 2.3, and since $\mathfrak{a}_{Y}^{0}=0$, we infer that $D_{X}^{2}=0$. Moreover, note that $\mathfrak{A}_{Y}^{0}$ is identically equal to 0 in the whole strip $\mathbb{R} \times \mathbb{T}$. Therefore, equality (40) leads to

$$
\sigma^{\sharp} \partial_{\tau} \mathfrak{A}_{Y}^{1}=\mathbb{1}_{\{0<X<f(\tau)\}}-\frac{q(X) / \sigma_{m}}{\int_{\mathbb{T}} 1 / \sigma^{\sharp} \mathrm{d} \tau} .
$$

Then, passing to the two-scale limit in (28), we infer that $D_{Y}^{2}$ converges to $D_{\infty}$, where

$$
D_{\infty}=\int_{0}^{+\infty}\left(\sigma_{m}-\sigma_{0}\right) \frac{q(X)(1-q(X))}{\sigma_{0} q(X)+\sigma_{m}(1-q(X))} \mathrm{d} X-\int_{\mathbb{T}} f(Y) \mathrm{d} Y
$$

The polarization tensor is then equal to

$$
\mathcal{M}_{\alpha}=\left(\begin{array}{cc}
-\int_{0}^{+\infty} q(s)\left(\sigma_{0} / \bar{\sigma}^{\sharp}(s)\right) \mathrm{d} s & 0 \\
0 & D_{\infty}
\end{array}\right)
$$

Remark 4.3. Note that since

we have

$$
\int_{0}^{+\infty} q(s) \mathrm{d} s=\int_{\mathbb{T}} f(Y) \mathrm{d} Y
$$

$$
\sigma_{0} \mathfrak{a}_{X}^{0}=\left(\sigma_{0}-\sigma_{m}\right) \int_{0}^{+\infty} \frac{q^{2}(s)}{\bar{\sigma}^{\sharp}(s)} \mathrm{d} s-\int_{\mathbb{T}} f(Y) \mathrm{d} Y .
$$

Hence, the formula for $\sigma_{0} \mathfrak{a}_{X}^{0}$ and the coefficient $\left(\sigma_{m}-\sigma_{0}\right) r_{1}-\tilde{f}$ of the polarization tensor given by Ciuperca $e t$ al. (Theorem 2.3, p. $6[13]$ ) coincide.

\section{Discussions}

We end this paper by linking our results to the results of Capdeboscq and Vogelius presented in Proposition 3 of [10], where the cases of thin parallel rectangle inhomogeneities for two different cases are discussed: the case of very oscillating inhomogeneities and the case of slowly oscillating domains. 


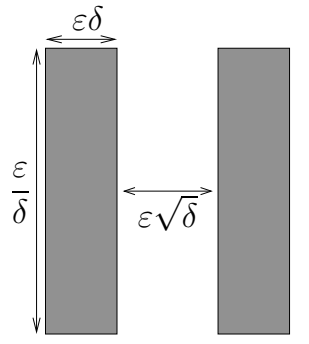

(a) $\delta=1 / 2$

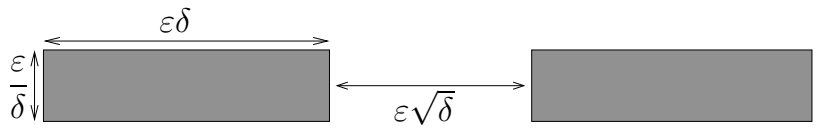

(b) $\delta=2$

FiguRE 8. Geometric framework of the inhomogeneity as described by Capdeboscq and Vogelius [10], $\varepsilon$ tending to zero and $\delta$ being a positive parameter such that the condition $\varepsilon<\min \left(\delta,(\delta+\sqrt{\delta})^{-1}\right.$ be satisfied.

For $\delta$ tending to zero, under the restrictive condition

$$
0<\varepsilon<\min \left(\delta,(\delta+\sqrt{\delta})^{-1},\right.
$$

the period of the oscillations is small, hence this describes the case of very thin vertical inclusions whose length is smaller than 1, while for $\delta$ tending to infinity the period of the oscillations tends to infinity and the inclusions are thin, horizontal, and of length 1.

The above geometry involve two small parameters $\epsilon$ and $\delta$, as described by figure 8. Moreover for $\delta$ tending to zero, the length does not necessarily tends to zero. Therefore, it is difficult to link straightforwardly the results of [10] to our case. However, we consider here two configurations that model similar problems.

\subsection{The case of rectangle inhomogeneity}

Let $\alpha>1$ : we are in the very oscillating case. Define the oscillating function $f_{\xi}$ on $\mathbb{T}$ for a given parameter $\xi \in(0,1)$ as follows:

$$
\forall \tau \in \mathbb{T}, \quad f_{\xi}(\tau)= \begin{cases}1, & \text { if } 0 \leq \tau \leq \sqrt{\xi} /(1+\sqrt{\xi}) \\ 0, & \text { elsewhere. }\end{cases}
$$

Observe that $f_{\xi}$ is not smooth, however as mentionned in the introduction, our result holds in this case after appropriate modifications. According to (45), the polarization tensor is then given by

$$
\mathcal{M}_{\alpha}=\frac{\sqrt{\xi}}{1+\sqrt{\xi}}\left(\begin{array}{cc}
M_{\nu}^{\xi} & 0 \\
0 & M_{\theta}^{\xi}
\end{array}\right) .
$$

where

$$
\begin{aligned}
& M_{\nu}^{\xi}=\left(1+\left(\sigma_{0}-\sigma_{m}\right) \frac{\sqrt{\xi}}{\sqrt{\xi} \sigma_{m}+\sigma_{0}}\right), \\
& M_{\theta}^{\xi}=\left(1+\left(\sigma_{0}-\sigma_{m}\right) \frac{1}{\sqrt{\xi} \sigma_{0}+\sigma_{m}}\right) .
\end{aligned}
$$

Passing to the limit for $\xi$ tending to zero, we deduce the formula

$$
\lim _{\xi \rightarrow 0} \mathcal{M}_{\alpha}=\left(\begin{array}{cc}
1 & 0 \\
0 & \sigma_{0} / \sigma_{m}
\end{array}\right)
$$


while for $\xi$ tending to infinity the polarization tensor is given by

$$
\lim _{\xi \rightarrow+\infty} M_{\alpha}=\left(\begin{array}{cc}
\sigma_{0} / \sigma_{m} & 0 \\
0 & 1
\end{array}\right)
$$

Observe that these formulae are similar to the results of Capdeboscq and Vogelius [10] in Proposition 3, since in their paper, $x_{1}$ is the tangential coordinate and $x_{2}$ is the normal coordinate, while here the first component refers to the normal coordinate and the second component to the tangential coordinate.

\subsection{Conclusion}

In this paper we have derived boundary layer correctors for the conductivity problem involving rough thin layers. We have provided a general framework that allows to treat simultaneously the cases of soft, rough and very rough thin layers, and we have shown error estimates that are valid in the whole domain. More particularly, our results lead to accurate description of the potential in the vicinity of the roughness. We also have explicitly described the generalized polarization tensor $\mathcal{M}_{\alpha}$. In particular, for $\alpha<1$, the polarization tensor is a diagonal matrix given by (34). For $\alpha=1$, the polarization tensor is a full $2 \times 2$ matrix, while for $\alpha>1$, it is diagonal again, but the matrix coefficients involve a mixture of the conductivities (see (45)). Numerical results have been published in [12].

\section{ACKNOWLEDGEMENTS}

The author thanks very warmly David Gérard-Varet from University Denis Diderot Paris 7 for his wellconsidered advice and suggestions, as well as the referees for providing constructive comments.

\section{REFERENCES}

[1] T. Abboud and H. Ammari. Diffraction at a curved grating: TM and TE cases, homogenization. J. Math. Anal. Appl., 202(3):995-1026, 1996.

[2] Y. Achdou and O. Pironneau. Domain decomposition and wall laws. C. R. Acad. Sci. Paris Sér. I Math., 320(5):541-547, 1995.

[3] G. Allaire and M. Amar. Boundary layer tails in periodic homogenization. ESAIM Control Optim. Calc. Var., 4:209-243 (electronic), 1999

[4] H. Ammari and H. Kang. Reconstruction of Small Inhomogeneities from Boundary Measurements. Lecture Notes in Mathematics, No. 1846. Springer-Verlag, Berlin, 2004.

[5] H. Ammari and H. Kang. Polarization and moment tensors, with applications to inverse problems and effective medium theory., volume 162 of Applied Mathematical Sciences. Springer, New York, 2007.

[6] A. Basson and D. Gérard-Varet. Wall laws for fluid flows at a boundary with random roughness. Comm. Pure Applied Math., $61(7), 2008$.

[7] E. Beretta and E. Francini. Asymptotic formulas for perturbations in the electromagnetic fields due to the presence of thin inhomogeneities. In Inverse problems: theory and applications (Cortona/Pisa, 2002), volume 333 of Contemp. Math., pages 49-62. Amer. Math. Soc., Providence, RI, 2003.

[8] E. Beretta, E. Francini, and M. S. Vogelius. Asymptotic formulas for steady state voltage potentials in the presence of thin inhomogeneities. A rigorous error analysis. J. Math. Pures Appl. (9), 82(10):1277-1301, 2003.

[9] Y. Capdeboscq and M. S. Vogelius. A general representation formula for boundary voltage perturbations caused by internal conductivity inhomogeneities of low volume fraction. M2AN Math. Model. Numer. Anal., 37(1):159-173, 2003.

[10] Y. Capdeboscq and M. S. Vogelius. Pointwise polarization tensor bounds, and applications to voltage perturbations caused by thin inhomogeneities. Asymptot. Anal., 50(3-4):175-204, 2006.

[11] I.S. Ciuperca, M. Jai, and C. Poignard. Approximate transmission conditions through a rough thin layer. The case of periodic roughness. Euro. Jnl of Applied Mathematics, 21(1):51-75, 2010.

[12] I.S. Ciuperca, R. Perrussel, and C. Poignard. Influence of a Rough Thin Layer on the Steady-state Potential. IEEE Trans. on Mag., 46(8), 2010.

[13] I.S. Ciuperca, R. Perrussel, and C. Poignard. Two-scale analysis for very rough thin layers. An explicit characterization of the polarization tensor. Journal de Mathématiques Pures et Appliquées, 95(3):277-295, 2011.

[14] W. Jäger, A. Mikelić, and N. Neuss. Asymptotic analysis of the laminar viscous flow over a porous bed. SIAM J. Sci. Comput., 22(6):2006-2028 (electronic), 2000. 
[15] V.A. Marchenko and E.Y. Khruslov. Homogenization of partial differential equations, volume 46 of Progress in Mathematical Physics. Birkhäuser Boston Inc., Boston, MA, 2006. Translated from the 2005 Russian original by M. Goncharenko and D. Shepelsky.

[16] C. Poignard. Approximate transmission conditions through a weakly oscillating thin layer. Math. Meth. App. Sci., 32(4):435453, 2009.

[17] C. Poignard, P. Dular, R. Perrussel, L. Krähenbühl, L. Nicolas, and M. Schatzman. Approximate conditions replacing thin layer. IEEE Trans. on Mag., 44(6):1154-1157, 2008. 